Sharif University of Technology
Scientia Iranica
Transactions B: Mechanical Engineering
IRAN I I IA

Research Note

\title{
Analytical solution to governing equations of triple coupled physics of structural mechanics, diffusion, and heat transfer
}

\author{
E. Mahdavi ${ }^{a}$, M. Haghighi-Yazdi ${ }^{a}$, M. Baniassadi ${ }^{a, b, *}$, M. Tehranic ${ }^{\text {c S. Ahzi }}$, \\ and J. Jamali \\ a. School of Mechanical Engineering, Faculty of Engineering, University of Tehran, Tehran, P.O. Box 11 55-4563, Iran. \\ b. University of Strasbourg, ICube laboratory-CNRS, 2 Rue Boussingault, 67000 Strasbourg, CS 10413, F-67412 Illkirch Cedex, \\ France. \\ c. Department of Mechanical Engineering, University of New Mexico, Albuquerque, NM 87131-0001, USA. \\ d. Qatar Environment and Energy Research Institute, Hamad Bin Khalifa University, Qatar Foundation, P.O. Box 5825, Qatar. \\ e. Department of Mechanical Engineering, Shoushtar Branch, Islamic Azad University, Shoushtar, P.O. Box 6451741117, Iran.
}

Received 10 June 2016; received in revised form 27 May 2017; accepted 30 April 2018

\section{KEYWORDS}

Fully coupled;

Diffusion;

Thermomechanical process;

Analytical solution;

Constitutive laws.

\begin{abstract}
Transport pipes have been widely used for their several advantages including their cost-effectiveness and simplicity of installation. These pipes are constantly in contact with the flowing fluid and, therefore, their material properties may degrade due to the diffusion of the fluid into the material system. These conditions are exacerbated as a result of high pressure and temperature of the transported fluid. Therefore, to simulate the behavior of such pipes, three interactive phenomena of mechanical stress, heat transfer, and mass diffusion need to be investigated. This study considers the three mechanisms simultaneously and provides an analytical solution to the corresponding coupled governing equations. The results of this work are in good agreement with the results of double coupled physics available in the literature and, therefore, can be used to predict the material behaviour under complicated environmental conditions.
\end{abstract}

(C) 2018 Sharif University of Technology. All rights reserved.

\section{Introduction}

Transportation and storing of refined or crude petroleum products, including oil and natural gas, have been carried out by pipeline systems and vessels for many years. This method ensures using a safe means of transporting fluids while providing a costeffective facility. Gas pipes and vessels are made

*. Corresponding author. Tel.: (0)3 68854554 E-mailaddress: m.baniassadi@ut.ac.ir (M. Baniassadi); mtehrani@unm.edu (M. Tehrani)

doi: $10.24200 /$ sci. 2018.20449 from steel coated with anti-corrosion materials or polymer materials that are usually corrosion resistant. The material system is usually designed to withstand different irreversible damage mechanisms caused by high temperature and pressurized fluid. Depending on the fluid-polymer system and operating thermal conditions, damage appears either locally [1] or in a diffuse way [1]. Because of the contact between fluid and the material system, the phenomenon of diffusion may also occur.

Diffusion can cause gradual degradation of material properties, but its effects become severer when coupled with mechanical loading and temperature variations. It is known that coupling between the three 
physics of diffusion, structural mechanics, and heat transfer makes the prediction of material's behavior more complicated. Therefore, without having a suitable model, it is difficult to analyze such problems and determine parameters such as interactions between the fluid and the material system, procedure of diffusion, and intergranular fracture. To understand the fully coupled thermal, mechanical, and diffusive mechanisms, it is essential to know constitutive laws and coupling mechanisms between the material and the fluid. The constitutive equations and interactions can also be described as a function of temperature, diffusant concentration, and fluid pressure using thermodynamic equations so that the material behavior is characterized.

There are various researches in open literature that involve coupled physics using two different approaches including theoretical and experimental studies. In both methods, a number of studies include dual coupled physics and some others include triple and quadruple problems.

For instance, in dual coupling studies, Vijalapura and Govindjee [2] proposed a coupled diffusiondeformation mechanism. Their research focused on numerical simulation of the mechanism by using the balance laws to develop partial differential equations and to solve these nonlinear equations using finite element and finite difference methods. Holalkere et al. [3] studied moisture and hygrothermal stress evaluated by fracture mechanics and finite element analysis on polymers. In their study, plastic package delamination was evaluated in order to obtain moisture sensitivity of plastic encapsulated microcircuits.

Govindjee and Simo [4] worked on the coupling which could happen in diffusive-mechanical problems and later, Busso [5] mentioned the interactions between mechanics and oxidation. Klopffer and Flaconneche [6] mentioned the diffusion process, which was a function of concentration. This phenomenon happens when a polymer material is plasticized by the penetrant. Teh et al. [7] described the effect of the coefficient of moisture expansion mismatch. They used finite element method to study this effect on hygroscopic swelling stress.

Abdel Wahab et al. [8] investigated the behavior of diffusion in adhesively bonded carbon fibre polymer composites. The research analyzed a nonlinear transient moisture problem using finite element model to evaluate moisture concentration as input data in stress analysis. Then, a comparison was made between analytical solution and the obtained results, which indicated that the thinner adhesive layer agreed well with the analytical solutions. Wong et al. [9] discussed finite element modeling in electronic multi-material packages with definite temperature and humidity as boundary conditions to solve the coupled diffusion and thermal processes. This simulation was performed based on experimental results.

Also, Yu and Pochiraju [10] studied the coupled phenomena in polymer composite materials considering temperature-dependent diffusion coefficients. Shirangi et al. [11] proposed another numerical method to obtain residual moisture content in modeling of components and IC packages.

Similarly, triple coupled physics were investigated by some authors such as Lin and Tay [12]. They used finite element method to predict delamination occurring in hygroscopic and thermal stresses in IC packages. Moreover, Yi and Sze [13] described moisture distribution and residual stress in IC packages, which were affected by temperature and moisture. Moreover, Chang et al. [14] explained moisture diffusion in plastic ball grid array packages while neglecting residual stresses. A similar study was done by Lahoti et al. [15] to conduct a three-dimensional finite element analysis of flip chip ball grid array packages. Viscoelastic behavior of polymeric components was studied by Dudek et al. [16]. Fan and Zhao [17] also proposed sequential coupled equations including moisture diffusion, heat conduction, and material deformation in encapsulated microelectronics devices. Their study was later extended by Rambert et al. [1] to develop a fully coupled mechanical-diffusion-thermal model.

There are also some experimental studies such as the works of Briscoe et al. [18,19] and Liatsis [20], which confirm the existence of a strong coupling between mechanical, thermal, and diffusion procedures. Some studies also include both theoretical and experimental investigations of the coupled physics, such as the recent work of Haghighi-Yazdi and Lee-Sullivan [21].

One of the most complete models studying triple coupled physics has been proposed by Rambert et al. [1] and includes the effective factors simultaneously. Rambert et al. [1] suggested a fully coupled mechanicaldiffusion-thermal model, which was developed based on finite element analysis. They used a Representative Volume Element (RVE) to illustrate thermo-diffusomechanical behavior of polymers. The considered RVE is the simplest one including a homogenous mixture of polymer and gas. This RVE is also explained in the thermodynamics of continuous media with internal variables. Therefore, the material characteristics have constant values. The considered RVE, however, does not include all sophisticated thermomechanics coupled with diffusion properties. Therefore, Rambert et al. [1] analyzed the problem in micro-scale, in which there exist different kinds of RVE depending on types of materials and considering how diffusion happens, as follows:

1. Monophasic modeling divided into three categories of Homogeneous Mixture, Saturated Porous 
Medium with an Impermeable Skeleton, and Porous Medium with a Permeable Skeleton. This is suitable for amorphous materials;

2. Biphasic modeling divided into two categories of Unsaturated Porous Medium with an Impermeable Skeleton and Unsaturated Porous Medium with a Permeable Skeleton. This is suitable for modeling the polymer mechanical behavior.

Moreover, this RVE is an open system and, thus, exchanges heat as well as matter with the surrounding medium. In addition, the material is assumed to be isotropic and is studied in the framework of small perturbations. Finally, a strong assumption, which consists in keeping each RVE characteristic constant in time and space, is made. To analyze this kind of situation means that thermo-diffuso-mechanical models describing micro and nanoscale are suitable [22]. Rambert and Grandidier [23] and Rambert et al. [1] extended the model further to include gradient-type elasticity. To the best of the authors' knowledge, there is no work in open literature on quadruple coupled physics.

Classical continuum mechanics has usually been employed by most researchers to develop the coupled physics. However, classical continuum mechanics can only describe bulk materials without considering surface effect, which can have significant influence on constitutive equations. Therefore, to consider the effect of surfaces in continuum mechanics, some additional parameters should be added to the conventional theorems of continuum mechanics. Moreover, considering surfaces plays a significant role in affecting the properties of the bulk material exposed to humid environments, which cause the initiation of diffusion and subsequent possible chemical reactions. To analyze the effect of surfaces and obtain constitutive laws, some investigations [24,25] have been conducted in different approaches to describe the surface effect based on Helmholtz energy. This energy is separated into two parts for the surface and the bulk material to explain their interactions [24,25]. A number of studies deal with multi-physics and describe their constitutive equations and material's responses. Different modeling approaches have been proposed, e.g., in the work of McBride et al. [22] who proposed a model at the micro- and nano-scales. This is while some other investigations tried to explain size-dependent response of materials, e.g., the works of Duan et al. [26], Miller and Shenoy [27], Mitrushchenkov et al. [28], Yang et al. [29], Wei et al. [30], and Yvonnet et al. [31].

Some studies considered material surface effects on the mechanical and thermal descriptions [32-35]. Gurtin and Murdoch [32] described surface stress in elastic media. They used kinematics and balance laws for the surface to explain tensorial nature of surface tension. This research was then extended by adding the thermal effect by Angenent and Gurtin [33]. Gurtin and Struthers [35] employed a novel description of surface effect to show the role of surface separating distance phases.

Daher and Maugin [36] proposed a method to obtain equations for interface in thermomechanical solid and, therefore, described complicated situations. Dolbow et al. [37] mentioned swelling of a hydrogel, which dealt with finite deformation. Their work was further extended through considering thermal effects by Ji and Gao [38]. Liu et al. [39] described adsorbate interactions in nanoscale and explained the mechanical properties of this phenomenon. Studies such as the work of Steinmann [40] continued further to include nonlinear continuum thermomechanics, diffusion, and viscoelasticity.

Moreover, the main purpose of such studies is to obtain governing equations to create a precise model. Therefore, it is required to have a model which is capable of representing three coupled equations including diffusion, mechanical stress-strain, and thermal effects.

As mentioned earlier, many investigations have been conducted to understand the mechanism of interaction of a fluid and the material system. There are, however, some restrictions to problem analysis due to the existence of coupling between mechanical and thermal physics. For example, Rodier-Renaud [41] proposed isobaric expansion coefficient as a function of temperature for polyethylene, but difficulty in doing tests makes such experimental study impossible. $\mathrm{Cu}-$ nat [42] considered direct coupling while taking into account specific free energy and internal dissipation phenomena such as mechanical, thermal, and diffusion mechanisms. Therefore, to model the behavior of a polymer exposed to a gaseous environment with high pressure and temperature, the interactions of three physical phenomena should be considered from modeling point of view. The interactions should include both direct and indirect coupling.

There are usually two kinds of methods employed in the open literature to analyze the problems involving dual, triple, and quadruple coupled physics: semicoupled analysis and fully coupled analysis. One of the studies which employed the fully coupled analysis method is the work of Rambert et al. [1]. However, the studies conducted by these authors are limited to numerical studies and, to the best of the authors' knowledge, there is no work in the open literature including the analytical solution to fully coupled problems. Therefore, a complete model is required, which can simultaneously include the effects of the three mechanisms on material properties as well as different interactions. Using mathematical method to solve the triple coupled equations can also provide a closed- 
form solution, which is preferable to numerical methods.

In this study, an analytical solution to the coupled mechanisms of diffusion, structural mechanics, and heat transfer is developed. The development of governing equations, which is based on the model proposed by Rambert et al. [1], is first explained. Next, an attempt is made to solve the equations analytically using Hankel transforms. The obtained closed-form solution is then used to investigate the effects of different coupling coefficients.

\section{Constitutive law and concepts}

To obtain an analytical solution to the full interaction between mechanical, thermal, and diffusion phenomena, some concepts need to be known about constitutive laws. These are described in many existing works, e.g., Fosdick and Tang [43], Gurtin and Murdoch [32], Gurtin and Struthers [35], and Steinmann [40]. Below, the two concepts of deformation and internal variables are briefly described.

\subsection{Deformation}

As mentioned before, it is important to consider the assumptions of both surface and bulk effects in a continuum, such as the one shown schematically in Figure 1 [22], which depicts material particles as point $\mathbf{X}$ that is related to body $\mathbf{B}_{\mathbf{0}}$. Also, the surface is denoted by $\mathbf{S}_{\mathbf{0}}$, and $\mathbf{N}$ shows the unit normal to $\mathbf{S}_{\mathbf{0}}$ (see [22] for further details).

Also, in order to map $d \mathbf{X}$ to $d x$, the following mapping function is introduced $[22,44]$. To define the mapping as derivative of the motion with respect to the reference placement, we use:

$$
F(X, t)=\operatorname{Grad} \phi(X, t),
$$

where the invertible tangent map $\mathbf{F}: T \beta_{0} \rightarrow T \beta_{t}$ (that is, the deformation gradient) maps a line element, $d \mathbf{X} \epsilon T \beta_{0}$, in the reference configuration to a line element, $d \mathbf{x} \in T \beta_{t}$, in the current configuration. The notation $T\{\boldsymbol{\square}\}$ denotes the tangent space to $\{\boldsymbol{\square}\}$. Also, $\operatorname{Grad}\{\boldsymbol{\square}\}=\partial\{\boldsymbol{\square}\} / \partial \mathbf{X}$ is the gradient operator with respect to the reference placement and $\operatorname{grad}\{\boldsymbol{\square}\}=$ $\partial\{\boldsymbol{G}\} / \partial \mathbf{x}$ refers to the current displacement (as shown in Figure 1). Therefore, to map other parameters such as the absolute temperature $T(\mathbf{X}, t)>0, \mathbf{F}(\mathbf{X}, t)$ maps Grad $\theta$ to $\operatorname{grad} T$ as follows $[22,44]$ :

$$
\text { Grad } T=F^{T} \operatorname{grad} T
$$

\subsection{Internal variables}

To include all parameters that may change during mechanical and chemical phenomena, the introduction of some internal variables is necessary to describe thermomechanical equations.

It is therefore assumed that there exists a function as follows [44]:

$$
\dot{\alpha}_{i}=f_{i}\left(T, F, \operatorname{grad} T, \alpha_{1}, \cdots \alpha_{N}\right), \quad i=1 \cdots N
$$

where $\boldsymbol{\alpha}$ is the internal state vector, $\alpha=\alpha(X, t)=$ $\left(\alpha_{1}, \alpha_{2}, \cdots, \alpha_{N}\right)$, and the number $\alpha_{i}=\alpha_{i}(X, t)$ is the internal state variables. Finding logical relation through thermodynamical conditions governed by Clausius-Duhem inequality between different parameters in Eq. (3) is important.

\section{Coupled triple phenomena}

As mentioned before, Rambert et al. [1] suggested a model that involved triple coupled physics and Rambert and Grandidier [23] extended this investigation, but they did not solve the equations analytically.

Moreover, the RVE was modeled with some characteristics such as RVE being assumed as an open system, which therefore, traded heat as well as matter with the surrounding medium. In addition, the material was assumed to be isotropic and was studied in the framework of small perturbations [45]. Finally, a strong assumption was considered by keeping each RVE characteristic constant with respect to time and

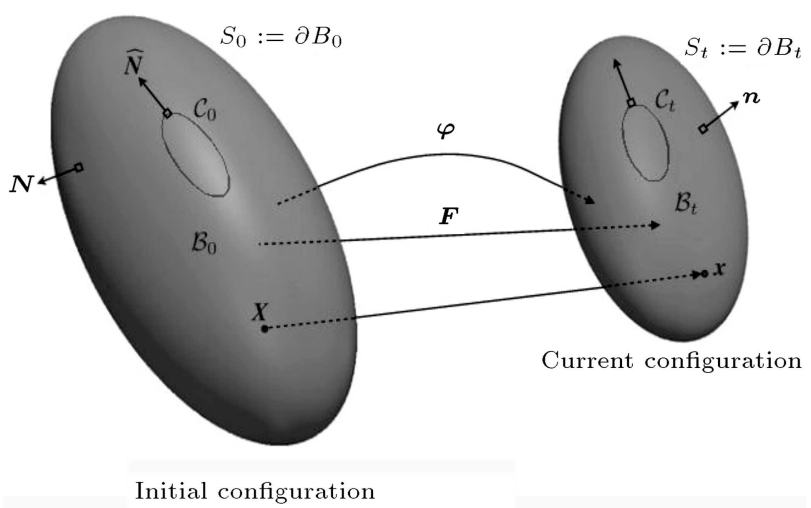

Figure 1. The motion $\varphi$ of the body from initial configuration to current configuration [22]. 
space. Fick's type law dominates diffusion, which is driven by concentration gradients.

Among the diffusion models described by Rambert et al. [1], a model was selected that implied complicated descriptions of the RVE by coupling three phenomena of diffusion, mechanical behavior, and thermal condition. The diffusion model was related to a semi-crystalline polymer as a porous medium. Some researchers such as Michaels and Bixler [46,47] proposed diffusion in this kind of material. Therefore, the mixture framework was chosen to develop the thermo-diffuso-mechanical model.

\section{Equation development}

To model the coupled equations using free and dissipative energy, internal variables representing the material are introduced according to Eq. (3). Some parameters such as stress, entropy, and chemical potential are thermodynamic forces; and strain, temperature, and normalized concentration are known as dual variables, which are linked by specific free energy. On the other hand, dissipative energy is employed to connect diffusion to thermal event.

\subsection{Multi-physics equations}

As mentioned before, there are three coupled equations, which involve the mechanical balance related to stress, heat balance linked with first and second laws of thermodynamics, and finally, mass balance related to mass diffusion.

\subsubsection{Mechanical balance}

The mechanical balance equation with some assumptions such as neglecting the inertia is derived from the fundamental principle of dynamics as follows:

$$
\operatorname{div} \sigma+\rho f=0
$$

where $\boldsymbol{\sigma}$ is the total Cauchy stress tensor, $f$ is the body force per unit of mass at any point within the RVE, $\operatorname{div}[\mathbf{\square}]$ denotes the divergence operator, and $\rho$ is average density of the mixture.

\subsubsection{Derivation of stress equilibrium equations}

The equilibrium equation by considering inertia effect is:

$$
\sigma_{i j, j}+\rho f_{i}=\rho \dot{v}_{i}
$$

For isotropic case, it can be obtained that:

$$
\begin{aligned}
\left(\sigma_{i j}\right)_{, j}= & (\lambda+\mu)\left(\frac{\partial^{2} u}{\partial r^{2}}-\frac{2}{r^{2}} u+\frac{2}{r} \frac{\partial u}{\partial r}\right) \\
& +\mu\left(\frac{\partial^{2} u}{\partial r^{2}}-\frac{2}{r^{2}} u+\frac{2}{r} \frac{\partial u}{\partial r}\right) .
\end{aligned}
$$

Assuming that changes are in radius direction in a spherical coordinate system and neglecting the body force, by substituting Eq. (6) into Eq. (5), the reduced constitutive equation of mechanical stress-strain is obtained:

$$
\begin{aligned}
\left(\frac{\partial^{2} u}{\partial r^{2}}\right. & \left.-\frac{2}{r^{2}} u+\frac{2}{r} \frac{\partial u}{\partial r}\right)-\frac{(3 \lambda+2 \mu)}{(\lambda+2 \mu)} \alpha_{T} \frac{\partial T}{\partial r} \\
& -\frac{(3 \lambda+2 \mu)}{(\lambda+2 \mu)} \alpha_{C} S_{g} \frac{\partial C}{\partial r}-\frac{1}{(\lambda+2 \mu)} \rho \ddot{u}_{i}=0
\end{aligned}
$$

\subsubsection{Thermal equation}

To solve the constitutive equation of mechanical stressstrain (Eq. (7)), the relation between displacement, $u$, concentration, $C$, and temperature, $T$, need to be known. To obtain the two variables of concentration and temperature, the two mechanisms of diffusion and heat conduction should be modeled according to Fick's and Fourier's laws, respectively. The derivation will be based on first and second laws of thermodynamics.

\subsubsection{Derivation of thermal equation}

According to Clausius-Duhem's in equality and the first law of thermodynamics, and by knowing that free energy can be changed by three parameters like displacement, concentration, and temperature, we have:

$$
\Omega=\Omega\left(\varepsilon_{i j}, \Delta C, \Delta T\right)
$$

Then, it can be written:

$$
\begin{aligned}
& T\left(\frac{\partial^{2} \Omega}{\partial \varepsilon_{i j} \partial T} \varepsilon_{i j}-\frac{\partial^{2} \Omega}{\partial T^{2}} T-\frac{\partial^{2} \Omega}{\partial C^{2}} C\right)-\rho Q+q_{i, i} \\
& \quad+[\Delta \mu+T d] q_{m g, i}=0 .
\end{aligned}
$$

For elastic behavior, the specific free energy, $\Omega$, of the mixture $(\mathrm{J} / \mathrm{kg})$ can be expressed as follows [1]:

$$
\Omega=\Omega\left(\varepsilon^{e}, C_{g}, C_{p}, T\right)
$$

where $T$ is temperature, $\varepsilon^{e}$ is the infinitesimal strain tensor, and the gas and polymer normalized concentrations are respectively denoted by $C_{g}$ and $C_{p}$. Furthermore, the second law of thermodynamics defines the volume dissipation related to entropy generation, and dissipation potential and its dual are coupled through Legendre-Fenchel transformation. Under the assumption of a total coupling between dissipative phenomena, the dual potential becomes a unique function [1]. When the dissipation phenomena are assumed to be driven by linear and fully coupled laws, the potential is quadratic and leads to an isotropic case with the following laws: 


$$
\begin{aligned}
q_{m g, i}= & \bar{J}_{m g}=-K_{\mu} \vec{D} s_{g} g r d C_{g} \\
& +K_{\mu} \frac{3 \lambda+2 \mu}{\rho} \operatorname{grad}\left[\operatorname{tr} \varepsilon^{e}\right] \\
& +\left(K_{\mu} \vec{d}-C_{T \mu}\right) g r d T \\
q_{i, i}= & \bar{J}_{q}=-\left(\left(K_{T} \vec{d}-C_{T \mu}\right) T\right. \\
+ & \left.\left(C_{T \mu}-\vec{d} K_{\mu}\right)\left(\mu_{g}-\mu_{p}\right)\right) \cdot \operatorname{grad} T \\
& -\frac{\vec{D} \rho}{K_{\mu}}\left(K_{\mu}\left(\mu_{g}-\mu_{p}\right)+C_{T \mu} T\right) s_{g} \operatorname{grad} C_{g} \\
& +\frac{(3 \lambda+2 \mu) \alpha_{C}}{\rho}\left(C_{T \mu} T+K_{\mu}\left(\mu_{g}-\mu_{p}\right)\right) \operatorname{grad}\left[\operatorname{tr} \varepsilon^{e}\right]
\end{aligned}
$$

where $k_{\mu}$ is linked to the chemical potential gradient effect on the gas mass flux, $k_{T}$ represents the thermal gradient effect on the entropy flux, and $C_{T_{\mu}}$ is a coupling coefficient corresponding to the temperature (chemical potential) gradient effect on the mass (entropy) flux.

Now, substituting the above equations into Eq. (9), we can have:

$$
\begin{aligned}
& \rho C_{V} \frac{d T}{d t}=\left(K_{T}+\left(K_{\mu} \vec{d}-2 C_{T \mu}\right) \vec{d}\right) \cdot T \cdot \operatorname{div}(\operatorname{grad} T) \\
& +r+\left(K_{\mu} \vec{d}-2 C_{T \mu}\right) \vec{d} \cdot(\operatorname{grad} T)^{2} \\
& +\frac{1}{K_{\mu}}\left(D \rho 10^{6} s_{g}\right)^{2}\left(\operatorname{grad} C_{g}\right)^{2}-(3 \lambda+2 \mu) \alpha_{T} T t r \frac{d \varepsilon^{e}}{d t} \\
& -2 D \rho 10^{6} s_{g} \frac{(3 \lambda+2 \mu)}{\rho} \alpha_{C} \operatorname{grad}\left[\operatorname{tr} \varepsilon^{e}\right] \cdot \operatorname{grad} C_{g} \\
& +K_{\mu}\left(\frac{(3 \lambda+2 \mu)}{\rho} \alpha_{C}\right)^{2}\left(\operatorname{grad}\left[\operatorname{tr} \varepsilon^{e}\right]\right)^{2} \\
& +\left(C_{T \mu}-K_{\mu} \vec{d}\right) \frac{1}{K_{\mu}} D \rho 10^{6} s_{g} T \operatorname{div}\left(\operatorname{grad} C_{g}\right) \\
& -\left(C_{T \mu}-K_{\mu} \vec{d}\right) T \frac{(3 \lambda+2 \mu)}{\rho} \alpha_{C} \operatorname{div}\left(\operatorname{grad}\left[\operatorname{tr} \varepsilon^{e}\right]\right) \\
& +2\left(C_{T \mu}-K_{\mu} \vec{d}\right) \frac{(3 \lambda+2 \mu)}{\rho} \alpha_{C}\left(\operatorname{grad}\left[\operatorname{tr} \varepsilon^{e}\right]\right) \cdot \operatorname{grad} T \\
& +2\left(C_{T \mu}-K_{\mu} \vec{d}\right) \frac{1}{K_{\mu}} D \rho 10^{6} s_{g} \operatorname{grad} C_{g} \cdot \operatorname{grad} T
\end{aligned}
$$

\subsubsection{Heat equation}

\subsubsection{Derivation of heat equation}

Assuming that there are two separate mass balance equations in the bi-component system leads to applying the classical balance equation to polymer and gas. For gas diffusion, we have:

$$
\rho . s_{g} \frac{d C_{g}}{d t}=-\operatorname{div} \bar{J}_{m g} \text {. }
$$

Obviously, the heat balance equation can be obtained [1]:

$$
\begin{aligned}
\rho C_{V} \frac{d T}{d t}= & -\operatorname{div} \vec{J}_{q}+r-(3 \lambda+2 \mu) \alpha_{T} T t r \frac{d \varepsilon^{e}}{d t} \\
& -\left[\left(\mu_{g}-\mu_{p}\right)+T \vec{d}\right] \cdot s_{g} \frac{d C_{g}}{d t}
\end{aligned}
$$

where $C_{V}=T\left(\frac{\partial S}{\partial T}\right)_{\varepsilon^{e}, C_{i}}$ is the specific heat at constant strain and normalized concentration, $\bar{J}_{q}$ is the heat flux per unit area, and $r$ is the volume density of heat generated by an external source. The last term in Eq. (15) corresponds to coupling between temperature variation and gas diffusion within the polymer material. By substituting the relation proposed by Rambert et al. [1] for $\mu_{g}-\mu_{p}$ in Eq. (15), we have:

$$
\begin{gathered}
\rho 10^{6} s_{g} \frac{d C_{g}}{d t}=D \rho 10^{6} s_{g} \operatorname{div}\left(\operatorname{grad} C_{g}\right) \\
+K_{\mu} \frac{(3 \lambda+2 \mu) \alpha_{C}}{\rho} \operatorname{grad}\left[\operatorname{tr} \varepsilon^{e}\right] \\
+\left(C_{T \mu}-K_{\mu} \vec{d}\right) \operatorname{div}(\operatorname{grad} T)
\end{gathered}
$$

\section{Analytical solution}

To develop a more straight-forward solution to the three coupled physics equations, a spherical coordinate system is assumed. Therefore, displacement, heat conduction, and mass transfer are only considered in radial direction, i.e., $u=u(r, t), T=T(r, t)$, and $C=C(r, t)$. Also, separate boundary conditions are employed for each boundary value problem. Cauchy and Dirichlet boundary conditions have been employed at the inner and outer surfaces. Thus, initial and boundary conditions for displacement, temperature, and concentration are, respectively, as follows:

Initial boundary condition for displacement:

$$
u(r, 0)=F_{1}(r), \quad \dot{u}(r, 0)=F_{2}(r) .
$$

Boundary conditions for displacement:

$$
\begin{aligned}
& \frac{\partial}{\partial r} u\left(R_{i}, 0\right)+k_{1} u\left(R_{i}, 0\right)=B_{1}(t), \\
& \frac{\partial}{\partial r} u\left(R_{o}, 0\right)+k_{2} u\left(R_{o}, 0\right)=B_{2}(t) .
\end{aligned}
$$


Initial boundary condition for temperature:

$$
T(r, 0)=F_{3}(r) .
$$

Boundary conditions for temperature:

$$
T\left(R_{i}, t\right)=g_{1}(t), \quad T\left(R_{o}, 0\right)=g_{2}(t) .
$$

Initial condition for concentration:

$$
C(r, 0)=F_{4}(r) .
$$

Boundary conditions for concentration:

$$
C\left(R_{i}, t\right)=f_{1}(t), \quad C\left(R_{o}, 0\right)=f_{2}(t) .
$$

Now, the following new variables are defined:

$$
\begin{aligned}
& u(r, t)=\frac{1}{\sqrt{r}} \omega(r, t), \\
& T(r, t)=\frac{1}{\sqrt{r}} \Phi(r, t), \\
& C(r, t)=\frac{1}{\sqrt{r}} \Psi(r, t) .
\end{aligned}
$$

By changing the variables in Eqs. (7), (13), and (16) to the newly defined variables above, the standard format of Hankel's equation will be obtained:

$$
\left.\begin{array}{rl}
\frac{\partial^{2} \omega}{\partial r^{2}}+ & \frac{1}{r} \frac{\partial \omega}{\partial r}-\frac{9}{4 r^{2}} \omega-\rho \frac{(1+v)(1-2 v)}{E(1-v)} \ddot{\omega} \\
& -\left(\frac{1+v}{1-v}\right) \alpha_{T}\left(\frac{\partial \varphi}{\partial r}-\frac{\varphi}{2 r}\right) \\
& -\left(\frac{1+v}{1-v}\right) s_{g} \alpha_{C}\left(\frac{\partial \psi}{\partial r}-\frac{\psi}{2 r}\right)=0 \\
\rho C_{V} & \frac{d \Phi}{d t}=\Lambda\left(\frac{\partial^{2} \Phi}{\partial r^{2}}+\frac{1}{r} \frac{\partial \Phi}{\partial r}-\frac{1}{4 r^{2}} \Phi\right) \\
& +\frac{A}{\sqrt{r}} \Phi\left(\frac{\partial^{2} \Phi}{\partial r^{2}}+\frac{1}{r} \frac{\partial \Phi}{\partial r}-\frac{1}{4 r^{2}} \Phi\right) \\
& +B \sqrt{r}\left(\frac{1}{\sqrt{r}} \frac{\partial \Phi}{\partial r}-\frac{1}{2} r^{-3 / 2} \Phi\right)^{2} \\
& \left(\frac{1}{\sqrt{r}} \frac{\partial \Psi}{\partial r}-\frac{1}{2} r^{-3 / 2} \Psi\right) \\
C & \sqrt{r}\left(\frac{1}{\sqrt{r}} \frac{\partial \Psi}{\partial r}-\frac{1}{2} r^{-3 / 2} \Psi\right)^{2} \\
- & D \Phi \frac{\partial}{\partial t}\left(\frac{1}{\sqrt{r}} \frac{\partial \omega}{\partial r}-\frac{1}{2} r^{-3 / 2} \omega\right) \\
\left.\sqrt{r} \frac{\partial^{2} \omega}{\partial r^{2}}-r^{-3 / 2} \frac{\partial \omega}{\partial r}+\frac{3}{4} r^{-5 / 2} \omega\right)
\end{array}\right)
$$

$$
\begin{aligned}
& +F \sqrt{r}\left(\frac{1}{\sqrt{r}} \frac{\partial^{2} \omega}{\partial r^{2}}-r^{-3 / 2} \frac{\partial \omega}{\partial r}+\frac{3}{4} r^{-5 / 2} \omega\right)^{2} \\
& +G \frac{1}{\sqrt{r}} \Phi\left(\frac{\partial^{2} \Psi}{\partial r^{2}}+\frac{1}{r} \frac{\partial \Psi}{\partial r}-\frac{1}{4 r^{2}} \Psi\right) \\
& -H \Phi\left(\frac{2}{r}\left(\frac{1}{\sqrt{r}} \frac{\partial^{2} \omega}{\partial r^{2}}-r^{-3 / 2} \frac{\partial \omega}{\partial r}+\frac{3}{4} r^{-5 / 2} \omega\right)\right) \\
& +\left(\frac{\partial}{\partial r}\right)\left(\frac{1}{\sqrt{r}} \frac{\partial^{2} \omega}{\partial r^{2}}-r^{-3 / 2} \frac{\partial \omega}{\partial r}+\frac{3}{4} r^{-5 / 2} \omega\right) \\
& +I \sqrt{r}\left(\frac{1}{\sqrt{r}} \frac{\partial \Psi}{\partial r}-\frac{1}{2} r^{-3 / 2} \Psi\right) \\
& .\left(\frac{1}{\sqrt{r}} \frac{\partial \Phi}{\partial r}-\frac{1}{2} r^{-3 / 2} \Phi\right) \\
& -J \sqrt{r}\left(\frac{1}{\sqrt{r}} \frac{\partial^{2} \omega}{\partial r^{2}}-r^{-3 / 2} \frac{\partial \omega}{\partial r}+\frac{3}{4} r^{-5 / 2} \omega\right) \\
& .\left(\frac{1}{\sqrt{r}} \frac{\partial \Phi}{\partial r}-\frac{1}{2} r^{-3 / 2} \Phi\right) .
\end{aligned}
$$

The coefficients in Eq. (20) can be calculated as follows:

$$
\begin{aligned}
& A=k_{T}+\left(k_{\mu} d-2 C_{T \mu}\right) d, \quad B=\left(k_{\mu} d-2 C_{T \mu}\right) d, \\
& r=0, \quad C=\frac{1}{k_{\mu}}\left(D \rho 10^{6} s_{g}\right)^{2}, \\
& D=(3 \lambda+2 \mu) \alpha_{T}, \quad E=2 D \rho 10^{6} s_{g} \frac{3 \lambda+2 \mu \alpha_{c}}{\rho}, \\
& F=k_{\mu}\left(\frac{(3 \lambda+2 \mu) \alpha_{c}}{\rho}\right)^{2} \text {, } \\
& G=\left(C_{T \mu}-k_{\mu} d\right) \frac{1}{k_{\mu}}\left(D \rho 10^{6} s_{g}\right), \\
& H=\left(C_{T \mu}-k_{\mu} d\right) \frac{(3 \lambda+2 \mu) \alpha_{c}}{\rho}, \\
& I=2\left(C_{T \mu}-k_{\mu} d\right) \frac{1}{k_{\mu}}\left(D \rho 10^{6} s_{g}\right), \\
& J=2\left(C_{T \mu}-k_{\mu} d\right) \frac{(3 \lambda+2 \mu) \alpha_{c}}{\rho}, \\
& \rho 10^{6} s_{g} \frac{d \Psi}{d t}=D \rho 10^{6} s_{g}\left(\frac{\partial^{2} \Psi}{\partial r^{2}}+\frac{1}{r} \frac{\partial \Psi}{\partial r}-\frac{1}{4 r^{2}} \Psi\right) \\
& +k_{\mu} \frac{(3 \lambda+2 \mu) \alpha_{C}}{\rho} \sqrt{r} \\
& \left(\left(\frac{2}{r}\left(\frac{1}{\sqrt{r}} \frac{\partial^{2} \omega}{\partial r^{2}}-r^{-3 / 2} \frac{\partial \omega}{\partial r}+\frac{3}{4} r^{-5 / 2} \omega\right)\right.\right.
\end{aligned}
$$




$$
\begin{aligned}
& \left.\left.+\frac{\partial}{\partial r}\left(\frac{1}{\sqrt{r}} \frac{\partial^{2} \omega}{\partial r^{2}}-r^{-3 / 2} \frac{\partial \omega}{\partial r}+\frac{3}{4} r^{-5 / 2} \omega\right)\right)\right) \\
& +\left(C_{T \mu}-k_{\mu} d\right)\left(\frac{\partial^{2} \Phi}{\partial r^{2}}+\frac{1}{r} \frac{\partial \Phi}{\partial r}-\frac{1}{4 r^{2}} \Phi\right)
\end{aligned}
$$

Also, using Eqs. (18a) to (18c) for boundary and initial conditions, the following relations are obtained:

Initial conditions:

$$
\begin{aligned}
& \omega(r, 0)=\sqrt{r} F_{1}(r), \\
& \Phi(r, 0)=\sqrt{r} F_{2}(r), \\
& \Psi(r, 0)=\sqrt{r} F_{4}(r) .
\end{aligned}
$$

Boundary conditions:

$$
\begin{aligned}
& \frac{\partial}{\partial r} \omega\left(R_{i}, 0\right)+k_{1}^{\prime} \omega\left(R_{i}, 0\right)=B_{1}^{\prime}(t), \\
& \frac{\partial}{\partial r} \omega\left(R_{o}, 0\right)+k_{2}^{\prime} \omega\left(R_{o}, 0\right)=B_{2}^{\prime}(t), \\
& \Phi\left(R_{i}, 0\right)=\sqrt{R_{i}} g_{1}(r), \\
& \Phi\left(R_{o}, 0\right)=\sqrt{R_{o}} g_{2}(r), \\
& \Psi\left(R_{i}, 0\right)=\sqrt{R_{i}} f_{1}(r), \\
& \Psi\left(R_{o}, 0\right)=\sqrt{R_{o}} f_{2}(r) .
\end{aligned}
$$

To solve the triple coupled Eqs. (19)-(21), the equations need to be divided into two parts, as follows:

$$
\begin{aligned}
& \omega=\omega_{1}+\omega_{2}, \\
& \Phi=\Phi_{1}+\Phi_{2}, \\
& \Psi=\Psi_{1}+\Psi_{2} .
\end{aligned}
$$

Then, the triple equations are divided into two parts and resolved into the following boundary value problems: The boundary value problem represented by Eq. (19), and related boundary and initial conditions are:

$$
\begin{aligned}
& \frac{\partial^{2} \omega_{1}}{\partial r^{2}}+\frac{1}{r} \frac{\partial \omega_{1}}{\partial r}-\frac{9}{4 r^{2}} \omega_{1}=\gamma^{2} \ddot{\omega}_{1} \\
& \frac{\partial}{\partial r} \omega_{1}\left(R_{i}, 0\right)+k_{1}^{\prime} \omega_{1}\left(R_{i}, 0\right)=B_{1}^{\prime}(t) \\
& \frac{\partial}{\partial r} \omega_{1}\left(R_{o}, 0\right)+k_{2}^{\prime} \omega_{1}\left(R_{o}, 0\right)=B_{2}^{\prime}(t), \\
& \omega_{1}(r, 0)=\dot{\omega}_{1}(r, 0)=0
\end{aligned}
$$

and:

$$
\begin{aligned}
& \left(\frac{\partial^{2} \omega_{2}}{\partial r^{2}}+\frac{1}{r} \frac{\partial \omega_{2}}{\partial r}-\frac{9}{4 r^{2}} \omega_{2}\right)-\rho \frac{(1+v)(1-2 v)}{E(1-v)} \omega_{2} \\
& -\frac{(3 \lambda+2 \mu)}{(\lambda+2 \mu)} \alpha_{T}\left(\frac{\partial \Phi}{\partial r}-\frac{\Phi}{2 r}\right) \\
& -\frac{(3 \lambda+2 \mu)}{(\lambda+2 \mu)} s_{g} \alpha_{C}\left(\frac{\partial \Psi}{\partial r}-\frac{\Psi}{2 r}\right)=0, \\
& \frac{\partial}{\partial r} \omega_{2}\left(R_{i}, 0\right)+k_{1}^{\prime} \omega_{2}\left(R_{i}, 0\right)=0, \\
& \frac{\partial}{\partial r} \omega_{2}\left(R_{o}, 0\right)+k_{2}^{\prime} \omega_{2}\left(R_{o}, 0\right)=0, \\
& \omega_{2}(r, 0)=\sqrt{r} F_{1}(r), \\
& \dot{\omega}_{2}(r, 0)=\sqrt{r} F_{2}(r) .
\end{aligned}
$$

Similarly, the boundary value problem of Eq. (20), and related boundary and initial conditions are:

$$
\begin{aligned}
& \left(\frac{\partial^{2} \Phi_{1}}{\partial r^{2}}+\frac{1}{r} \frac{\partial \Phi_{1}}{\partial r}-\frac{1}{4 r^{2}} \Phi_{1}\right)=\frac{\rho C_{V}}{\Lambda} \frac{d \Phi_{1}}{d t}, \\
& \Phi_{1}\left(R_{i}, t\right)=\sqrt{R_{i}} g_{1}(t), \\
& \Phi_{1}\left(R_{o}, t\right)=\sqrt{R_{o}} g_{2}(t), \\
& \Phi_{1}(r, 0)=0
\end{aligned}
$$

and:

$$
\begin{aligned}
\left(\frac{\partial^{2} \Phi_{2}}{\partial r^{2}}\right. & \left.+\frac{1}{r} \frac{\partial \Phi_{2}}{\partial r}-\frac{1}{4 r^{2}} \Phi_{2}\right)-\frac{\rho C_{V}}{\Lambda} \frac{d \Phi_{2}}{d t} \\
= & Z\left(r, \Phi_{2}, \Psi, \omega\right), \\
\Phi_{2}\left(R_{i}, t\right) & =0 \\
\Phi_{2}\left(R_{o}, t\right) & =0 \\
\Phi_{2}(r, 0) & =\sqrt{r} F_{3}(r) .
\end{aligned}
$$

Also, the boundary value problem of Eq. (21), and related boundary and initial conditions are written as:

$$
\begin{aligned}
& \frac{\partial^{2} \Psi_{1}}{\partial r^{2}}+\frac{1}{r} \frac{\partial \Psi_{1}}{\partial r}-\frac{1}{4 r^{2}} \Psi_{1}-\frac{1}{D} \frac{d \Psi_{1}}{d t}=0 \\
& \Psi_{1}\left(R_{i}, t\right)=\sqrt{R_{i}} f_{1}(t) \\
& \Psi_{1}\left(R_{o}, t\right)=\sqrt{R_{o}} f_{2}(t) \\
& \Psi_{1}(r, 0)=0
\end{aligned}
$$


and:

$$
\begin{aligned}
& \frac{\partial^{2} \Psi_{2}}{\partial r^{2}}+\frac{1}{r} \frac{\partial \Psi_{2}}{\partial r}-\frac{1}{4 r^{2}} \Psi_{2}-\frac{1}{D} \frac{d \Psi_{2}}{d t}=Z Z(r, \omega, \Phi), \\
& \Psi_{2}\left(R_{i}, t\right)=0 \\
& \Psi_{2}\left(R_{o}, t\right)=0 \\
& \Psi_{2}(r, t)=\sqrt{r} F_{4}(r) .
\end{aligned}
$$

Eqs. (25a), (27a), and (29a) are of Bessel type and can be solved by finite Hankel transformation [48]. According to boundary conditions for boundary value problems, i.e., Eqs. (25), (27), and (29), the kernels can be written as [48]:

$$
\begin{aligned}
& K_{1}\left(r, \lambda_{n}\right) \\
& =J_{1.5}\left(\lambda_{n} r\right)\left(\lambda_{n} \frac{d}{d r} Y_{1.5}\left(\lambda_{n} R_{i}\right)+k_{1}^{\prime} Y_{1.5}\left(\lambda_{n} R_{i}\right)\right) \\
& -Y_{1.5}\left(\lambda_{n} r\right)\left(\lambda_{n} \frac{d}{d r} J_{1.5}\left(\lambda_{n} R_{i}\right)+k_{1}^{\prime} J_{1.5}\left(\lambda_{n} R_{i}\right)\right) \\
& K_{2}\left(r, \zeta_{m}\right)=J_{0.5}\left(r \zeta_{m}\right) Y_{0.5}\left(R_{i} \zeta_{m}\right) \\
& \quad-J_{0.5}\left(R_{i} \zeta_{m}\right) Y_{0.5}\left(r \zeta_{m}\right) \\
& K_{3}\left(r, \gamma_{p}\right)=J_{0.5}\left(r \gamma_{p}\right) Y_{0.5}\left(R_{i} \gamma_{p}\right) \\
& -J_{0.5}\left(R_{i} \gamma_{p}\right) Y_{0.5}\left(r \gamma_{p}\right) .
\end{aligned}
$$
below:

$\lambda_{n}, \xi_{m}$, and $\gamma_{p}$ are positive roots of the equations

$$
\begin{gathered}
\left(\frac{d}{d r} Y_{1.5}\left(\lambda_{n} R_{i}\right)+k_{1}^{\prime} Y_{1.5}\left(\lambda_{n} R_{i}\right)\right) \\
\quad\left(\frac{d}{d r} J_{1.5}\left(\lambda_{n} R_{o}\right)+k_{2}^{\prime} J_{1.5}\left(\lambda_{n} R_{o}\right)\right) \\
=\left(\frac{d}{d r} Y_{1.5}\left(\lambda_{n} R_{o}\right)+k_{2}^{\prime} Y_{1.5}\left(\lambda_{n} R_{o}\right)\right) \\
\quad\left(\frac{d}{d r} J_{1.5}\left(\lambda_{n} R_{i}\right)+k_{1}^{\prime} J_{1.5}\left(\lambda_{n} R_{i}\right)\right), \\
J_{0.5}\left(\zeta_{m} R_{i}\right) Y_{0.5}\left(\zeta_{m} R_{o}\right)-J_{0.5}\left(\zeta_{m} R_{o}\right) Y_{0.5}\left(\zeta_{m} R_{i}\right)=0, \\
J_{0.5}\left(\gamma_{p} R_{i}\right) Y_{0.5}\left(\gamma_{p} R_{o}\right)-J_{0.5}\left(\gamma_{p} R_{o}\right) Y_{0.5}\left(\gamma_{p} R_{i}\right)=0 .
\end{gathered}
$$

Now, using Hankel transformation in Eqs. (25a), (27a), and (29a), we have:

$$
\begin{aligned}
& H\left\{\frac{1}{\gamma^{2}}\left(\frac{\partial^{2} \omega_{1}}{\partial r^{2}}+\frac{1}{r} \frac{\partial \omega_{1}}{\partial r}-\frac{9}{4 r^{2}} \omega_{1}\right)-\ddot{\omega}\right\} \\
& =\frac{2\left(\lambda_{n} \frac{d}{d r} J_{1.5}\left(\lambda_{n} R_{i}\right)+k_{1}^{\prime} J_{1.5}\left(\lambda_{n} R_{i}\right)\right)}{\gamma^{2}\left(\lambda_{n} \frac{d}{d r} J_{1.5}\left(\lambda_{n} R_{o}\right)+k_{2}^{\prime} J_{1.5}\left(\lambda_{n} R_{o}\right)\right)} B_{2}^{\prime}(t) \\
& -\frac{2}{\gamma^{2} \pi} B_{1}^{\prime}(t) \\
& H\left\{\frac{\Lambda}{\rho C_{V}}\left(\frac{\partial^{2} \Phi_{1}}{\partial r^{2}}+\frac{1}{r} \frac{\partial \Phi_{1}}{\partial r}-\frac{1}{4 r^{2}} \Phi_{1}\right)-\frac{d \Phi_{1}}{d t}\right\} \\
& =\frac{\Lambda}{\rho C_{V}}\left(\frac{2 J_{0.5}\left(\zeta_{m} R_{i}\right)}{\pi J_{0.5}\left(\zeta_{m} R_{o}\right)} \sqrt{R_{0}} g_{2}(t)-\frac{2}{\pi} \sqrt{R_{i}} g_{1}(t)\right), \\
& H\left\{\frac{\partial^{2} \Psi_{1}}{\partial r^{2}}+\frac{1}{r} \frac{\partial \Psi_{1}}{\partial r}-\frac{1}{4 r^{2}} \Psi_{1}-\frac{1}{D} \frac{d \Psi_{1}}{d t}\right\} \\
& =\left(\frac{2 J_{0.5}\left(\gamma_{p} R_{i}\right)}{\pi J_{0.5}\left(\gamma_{p} R_{o}\right)} \sqrt{R_{0}} f_{2}(t)-\frac{2}{\pi} \sqrt{R_{i}} f_{1}(t)\right) .
\end{aligned}
$$

Now, the following equations are obtained:

$$
\begin{aligned}
& \frac{\lambda_{n}^{2}}{\gamma^{2}} \bar{\omega}_{1}\left(t, \lambda_{n}\right)+\frac{\partial^{2} \bar{\omega}_{1}\left(t, \lambda_{n}\right)}{\partial t^{2}} \\
& \quad=\frac{2\left(\lambda_{n} \frac{d}{d r} J_{1.5}\left(\lambda_{n} R_{i}\right)+k_{1}^{\prime} J_{1.5}\left(\lambda_{n} R_{i}\right)\right)}{\gamma^{2} \pi\left(\lambda_{n} \frac{d}{d r} J_{1.5}\left(\lambda_{n} R_{o}\right)+k_{2}^{\prime} J_{1.5}\left(\lambda_{n} R_{o}\right)\right)} B_{2}^{\prime}(t) \\
& \quad-\frac{2}{\gamma^{2} \pi} B_{1}^{\prime}(t)
\end{aligned}
$$

$$
\begin{aligned}
& \frac{\Lambda}{\rho C_{V}} \zeta_{m}^{2} \bar{\Phi}_{1}\left(t, \zeta_{m}\right)+\frac{d \bar{\Phi}_{1}\left(t, \zeta_{m}\right)}{d t} \\
& \quad=\frac{\Lambda}{\rho C_{V}}\left(\frac{2 J_{0.5}\left(\zeta_{m} R_{i}\right)}{\pi J_{0.5}\left(\zeta_{m} R_{o}\right)} \sqrt{R_{o}} g_{2}(t)-\frac{2}{\pi} \sqrt{R_{i}} g_{1}(t)\right), \\
& D \gamma_{p}^{2} \bar{\Psi}_{1}\left(t, \gamma_{p}\right)+\frac{d \bar{\Psi}_{1}\left(t, \gamma_{p}\right)}{d t} \\
& \quad=D\left(\frac{2 J_{0.5}\left(\gamma_{p} R_{i}\right)}{\pi J_{0.5}\left(\gamma_{p} R_{o}\right)} \sqrt{R_{o}} f_{2}(t)-\frac{2}{\pi} \sqrt{R_{i}} f_{1}(t)\right)
\end{aligned}
$$

Using Laplace transformation leads to the following:

$$
\bar{\omega}_{1}\left(t, \lambda_{n}\right)=L^{-1}\left\{\frac{\Gamma(S)}{S^{2}+\frac{\lambda_{n}^{2}}{\gamma^{2}}}\right\},
$$

$\bar{\Phi}_{1}\left(t, \zeta_{m}\right)=L^{-1}\left\{\frac{\Pi(S)}{S^{2}+\frac{\Lambda}{\rho C_{V}} \zeta_{m}^{2}}\right\}$,

$$
\bar{\Psi}_{1}\left(t, \gamma_{p}\right)=L^{-1}\left\{\frac{\Omega(S)}{S^{2}+D \gamma_{p}^{2}}\right\},
$$


where:

$$
\begin{aligned}
\Gamma(t) & =\frac{2\left(\lambda_{n} \frac{d}{d r} J_{1.5}\left(\lambda_{n} R_{i}\right)+k_{1}^{\prime} J_{1.5}\left(\lambda_{n} R_{i}\right)\right)}{\gamma^{2} \pi\left(\lambda_{n} \frac{d}{d r} J_{1.5}\left(\lambda_{n} R_{o}\right)+k_{2}^{\prime} J_{1.5}\left(\lambda_{n} R_{o}\right)\right)} B_{2}^{\prime}(t) \\
& -\frac{2}{\gamma^{2} \pi} B_{1}^{\prime}(t),
\end{aligned}
$$$$
\Pi(t)=\frac{\Lambda}{\rho C_{V}}\left(\frac{2 J_{0.5}\left(\zeta_{m} R_{i}\right)}{\pi J_{0.5}\left(\zeta_{m} R_{o}\right)} \sqrt{R_{o}} g_{2}(t)-\frac{2}{\pi} \sqrt{R_{i}} g_{1}(t)\right),
$$$$
\Omega(t)=D\left(\frac{2 J_{0.5}\left(\gamma_{p} R_{i}\right)}{\pi J_{0.5}\left(\gamma_{p} R_{o}\right)} \sqrt{R_{o}} f_{2}(t)-\frac{2}{\pi} \sqrt{R_{i}} f_{1}(t)\right)
$$

The inverse finite Hankel transform is obtained by Eqs. 36(a) to 36(c), as shown in Box I. Finally, we have:

$$
\begin{aligned}
\omega_{1}(r, t)= & \sum_{\lambda_{n}} a_{n} K_{1}\left(r, \lambda_{n}\right) \bar{\omega}_{1}\left(t, \lambda_{n}\right) \\
= & \sum_{\lambda_{n}}\left(a _ { n } J _ { 1 . 5 } ( \lambda _ { n } r ) \left(\lambda_{n} \frac{d}{d r} Y_{1.5}\left(\lambda_{n} R_{i}\right)\right.\right. \\
& \left.+k_{1}^{\prime} Y_{1.5}\left(\lambda_{n} R_{i}\right)\right) \\
& -Y_{1.5}\left(\lambda_{n} r\right)\left(\lambda_{n} \frac{d}{d r} J_{1.5}\left(\lambda_{n} R_{i}\right)\right.
\end{aligned}
$$

$$
\begin{gathered}
\left.\left.+k_{1}^{\prime} J_{1.5}\left(\lambda_{n} R_{i}\right)\right)\right) \cdot L^{-1}\left\{\frac{\Gamma(S)}{S^{2}+\frac{\lambda_{n}^{2}}{\gamma^{2}}}\right\} \\
\Phi_{1}(r, t)=\sum_{\zeta_{m}} b_{m} K_{2}\left(r, \zeta_{m}\right) \bar{\Phi}_{1}\left(t, \zeta_{m}\right) \\
=\frac{\pi^{2}}{2} \sum_{\lambda_{n}}\left(\frac{\zeta_{m}^{2} J_{0.5}^{2}\left(\zeta_{m} R_{i}\right)}{J_{0.5}^{2}\left(\zeta_{m} R_{i}\right)-J_{0.5}^{2}\left(\zeta_{m} R_{o}\right)}\right) \\
J_{0.5}\left(r \zeta_{m}\right) Y_{0.5}\left(R_{i} \zeta_{m}\right)-J_{0.5}\left(R_{i} \zeta_{m}\right) Y_{0.5}\left(r \zeta_{m}\right) \\
\left.. L^{-1}\left\{\frac{\Pi(S)}{\Psi_{1}^{2}+\frac{\Lambda}{\rho C_{V}} \zeta_{m}^{2}}\right\}, \gamma_{p}\right)=\sum_{\gamma_{p}} E_{p} K_{3}\left(r, \gamma_{p}\right) \bar{\Psi}_{1}\left(t, \gamma_{p}\right) \\
=\frac{\pi^{2}}{2} \sum_{\lambda_{n}}\left(\frac{\gamma_{p}^{2} J_{0.5}^{2}\left(\gamma_{p} R_{i}\right)}{J_{0.5}^{2}\left(\gamma_{p} R_{i}\right)-J_{0.5}^{2}\left(\gamma_{p} R_{o}\right)}\right) \\
J_{0.5}\left(r \gamma_{p}\right) Y_{0.5}\left(R_{i} \gamma_{p}\right)-J_{0.5}\left(R_{i} \gamma_{p}\right) Y_{0.5}\left(r \gamma_{p}\right) \\
\left.\frac{\Omega(S)}{S^{2}+D \gamma_{p}^{2}}\right\} \cdot
\end{gathered}
$$

The inverse finite Hankel transform is as follows:

$$
\begin{aligned}
& \omega_{1}(r, t)=\sum_{\lambda_{n}} a_{n} K_{1}\left(r, \lambda_{n}\right) \bar{\omega}_{1}\left(t, \lambda_{n}\right), \\
& \Phi_{1}(r, t)=\sum_{\zeta_{m}} b_{m} K_{2}\left(r, \zeta_{m}\right) \bar{\Phi}_{1}\left(t, \zeta_{m}\right),
\end{aligned}
$$

$$
\begin{aligned}
\omega_{1}(r, t)= & \sum_{\lambda_{n}} \frac{1}{\int_{R_{i}} r\left(K_{1}\left(r, \lambda_{n}\right)\right)^{2} d r} \\
= & \frac{\lambda_{n}^{2} \pi^{2}\left(\lambda_{n} \frac{d}{d r} J_{1.5}\left(\lambda_{n} R_{o}\right)+k_{2}^{\prime} J_{1.5}\left(\lambda_{n} R_{o}\right)\right)^{2}}{\left({k_{2}^{\prime}}_{2}^{2}+\lambda_{n}^{2}\left(1-\left(\frac{1.5}{\lambda_{n} R_{o}}\right)^{2}\right)\right)\left(\left(\lambda_{n} \frac{d}{d r} J_{1.5}\left(\lambda_{n} R_{i}\right)+k_{1}^{\prime} J_{1.5}\left(\lambda_{n} R_{i}\right)\right)^{2}\right)} K_{1}\left(r, \lambda_{n}\right) \bar{\omega}_{1}\left(t, \lambda_{n}\right) . \\
\Phi_{1}(r, t)= & \frac{\pi^{2}}{2} \sum_{\zeta_{m}}\left(\frac{\zeta_{m}^{2} J_{0.5}^{2}\left(\zeta_{m} R_{i}\right)}{J_{0.5}^{2}\left(\zeta_{m} R_{i}\right)-J_{0.5}^{2}\left(\zeta_{m} R_{o}\right)}\right) K_{2}\left(r, \zeta_{m}\right) \bar{\Phi}_{1}\left(t, \zeta_{m}\right), \\
\Psi_{1}(r, t)= & \frac{\pi^{2}}{2} \sum_{\gamma_{p}}\left(\frac{\gamma_{p}^{2} J_{0.5}^{2}\left(\gamma_{p} R_{i}\right)}{J_{0.5}^{2}\left(\gamma_{p} R_{i}\right)-J_{0.5}^{2}\left(\gamma_{p} R_{o}\right)}\right) K_{3}\left(r, \gamma_{p}\right) \bar{\Psi}_{1}\left(t, \gamma_{p}\right),
\end{aligned}
$$




$$
\Psi_{1}\left(r, \gamma_{p}\right)=\sum_{\gamma_{p}} E_{p} K_{3}\left(r, \gamma_{p}\right) \bar{\Psi}_{1}\left(t, \gamma_{p}\right)
$$

Therefore, Eqs. (25a), (27a), and (29a) are solved, which lead, respectively, to Eqs. (38a) to (38c). Next, the solution to Eqs. (26a) to (30a) will be developed.

First, the following relations, which can be easily shown to satisfy the boundary conditions in Eqs. (26b), $(28 \mathrm{~b})$, and (30b), are assumed:

$$
\begin{aligned}
& \omega_{2}=S(t) K_{1}\left(r, \lambda_{n}\right), \\
& \Phi_{2}=P(t) K_{2}\left(r, \zeta_{m}\right), \\
& \Psi_{2}=X(t) K_{3}\left(r, \gamma_{p}\right) .
\end{aligned}
$$

$S(t), P(t)$, and $X(t)$ are unknown time-dependent functions that need to be obtained. By substituting Eq. (33a) to Eq. (33c) into Eqs. (26a), (28a), and (30a), we have:

$$
\begin{aligned}
&(\ddot{S}(t)\left.+\left(\frac{\lambda_{n}}{\gamma}\right)^{2} S(t)\right) K_{1}\left(r, \lambda_{n}\right) \\
&=-\frac{1}{\gamma^{2}}\left(\frac{3 \lambda+2 \mu}{\lambda+2 \mu}\right) \alpha_{T}\left(b_{m} \bar{\Phi}_{1}+P(t)\right) \\
&\left(\frac{d K_{2}\left(r, \xi_{m}\right)}{d r}-\frac{K_{2}\left(r, \xi_{m}\right)}{2 r}\right) \\
&-\frac{1}{\gamma^{2}}\left(\frac{3 \lambda+2 \mu}{\lambda+2 \mu}\right) s_{g} \alpha_{C}\left(E_{p} \bar{\Psi}_{1}+X(t)\right) \\
&\left(\frac{d K_{3}\left(r, \gamma_{p}\right)}{d r}-\frac{K_{3}\left(r, \gamma_{p}\right)}{2 r}\right) \\
&\left(\frac{\Lambda}{\rho C_{V}} \zeta_{m}^{2} P(t)+\dot{P}(t)\right) K_{2}\left(r, \zeta_{m}\right) \\
&=W W\left(P, S, t, r, \zeta_{m}, \gamma_{p}, \lambda_{n}\right) \\
&\left(D \gamma_{p}^{2} F(t)+\dot{F}(t)\right) K_{3}\left(r, \gamma_{p}\right) \\
&=W W W\left(P, S, t, r, \zeta_{m}, \gamma_{p}, \lambda_{n}\right)
\end{aligned}
$$
where:

$$
\begin{aligned}
W W\left(P, S, t, r, \zeta_{m}, \gamma_{p}, \lambda_{n}\right) & \\
= & \frac{\Lambda}{\rho C} P(t)^{2} K_{2}\left(r, \zeta_{m}\right)\left(\frac{1}{\sqrt{r}} \frac{d^{2} K_{2}\left(r, \zeta_{m}\right)}{d r^{2}}\right. \\
& \left.+r^{-3 / 2} \frac{d K_{2}\left(r, \zeta_{m}\right)}{d r}-\frac{1}{4} r^{-5 / 2} K_{2}\left(r, \zeta_{m}\right)\right)
\end{aligned}
$$

$$
\begin{aligned}
& -\frac{B}{\rho C} P(t)\left(\frac{d K_{2}\left(r, \zeta_{m}\right)}{d r}-\frac{1}{2} r^{-1} K_{2}\left(r, \zeta_{m}\right)\right)^{2} \\
& +\frac{1}{\rho C} P(t)\left(\frac{d K_{2}\left(r, \zeta_{m}\right)}{d r}-\frac{1}{2} r^{-1} K_{2}\left(r, \zeta_{m}\right)\right) \\
& \left(-I\left(E_{p} \bar{\Psi}_{1}\left(t, \gamma_{p}\right)\right.\right. \\
& +X(t)\left(\frac{1}{\sqrt{r}} \frac{d K_{3}\left(r, \gamma_{p}\right)}{d r} \frac{1}{2} r^{-3 / 2} K_{3}\left(r, \gamma_{p}\right)\right) \\
& +J\left(a_{n} \bar{\omega}_{1}\left(t, \lambda_{n}\right)+S(t)\right)\left(\frac{1}{\sqrt{r}} \frac{d^{2} K_{1}\left(r, \lambda_{n}\right)}{d r^{2}}\right. \\
& \left.\left.-r^{-3 / 2} \frac{d K_{1}\left(r, \lambda_{n}\right)}{d r}+\frac{3}{4} r^{-5 / 2} K_{1}\left(r, \lambda_{n}\right)\right)\right) \\
& =\frac{1}{\rho C} C\left(( E _ { p } \overline { \Psi } _ { 1 } ( t , \gamma _ { p } ) + X ( t ) ) \left(\frac{d K_{3}\left(r, \gamma_{p}\right)}{d r}\right.\right. \\
& \left.\left.-\frac{1}{2} r^{-1} K_{3}\left(r, \gamma_{p}\right)\right)\right)^{2}-\frac{1}{\rho C} D P(t) K_{2}\left(r, \zeta_{m}\right) \\
& \cdot\left(a_{n} \dot{\bar{\omega}}_{1}\left(t, \lambda_{n}\right)+\dot{S}(t)\right)\left(\frac{1}{\sqrt{r}} \frac{d K_{1}\left(r, \lambda_{n}\right)}{d r}\right. \\
& \left.-\frac{1}{2} r^{-3 / 2} K_{1}\left(r, \lambda_{n}\right)\right) \\
& -\frac{1}{\rho C} E\left(a_{n} \bar{\omega}_{1}\left(t, \lambda_{n}\right)+S(t)\right)^{2}\left(\frac{d^{2} K_{1}\left(r, \lambda_{n}\right)}{d r^{2}}\right. \\
& \left.-r^{-1} \frac{d K_{1}\left(r, \lambda_{n}\right)}{d r}+\frac{3}{4} r^{-2} K_{1}\left(r, \lambda_{n}\right)\right) \\
& \cdot\left(\frac{1}{\sqrt{r}} \frac{d K_{1}\left(r, \lambda_{n}\right)}{d r}-\frac{1}{2} r^{-3 / 2} K_{1}\left(r, \lambda_{n}\right)\right) \\
& +\frac{1}{\rho C} F \sqrt{r}\left(a_{n} \bar{\omega}_{1}\left(t, \lambda_{n}\right)+S(t)\right)^{2}
\end{aligned}
$$$$
\cdot\left(\frac{1}{\sqrt{r}} \frac{d^{2} K_{1}\left(r, \lambda_{n}\right)}{d r^{2}}-r^{-3 / 2} \frac{d K_{1}\left(r, \lambda_{n}\right)}{d r}\right.
$$$$
\left.+\frac{3}{4} r^{-5 / 2} K_{1}\left(r, \lambda_{n}\right)\right)^{2}
$$$$
+\frac{1}{\rho C} G \frac{1}{\sqrt{r}} P(t) K_{2}\left(r, \zeta_{m}\right)
$$ 


$$
\begin{aligned}
& \cdot\left(X(t)+E_{p} \bar{\Psi}_{1}\left(t, \gamma_{p}\right)\right)\left(\frac{d^{2} K_{3}\left(r, \gamma_{p}\right)}{d r^{2}}\right. \\
& \left.+\frac{1}{r} \frac{d K_{3}\left(r, \gamma_{p}\right)}{d r} \frac{1}{4 r^{2}} K_{3}\left(r, \gamma_{p}\right)\right) \\
& +\frac{1}{\rho C} H P(t) K_{2}\left(r, \zeta_{m}\right)\left(a_{n} \bar{\omega}_{1}\left(t, \lambda_{n}\right)+S(t)\right) \\
& \cdot\left(\frac { 2 } { r } \left(\frac{1}{\sqrt{r}} \frac{d^{2} K_{1}\left(r, \lambda_{n}\right)}{d r^{2}}-r^{-3 / 2} \frac{d K_{1}\left(r, \lambda_{n}\right)}{d r}\right.\right. \\
& \left.+\frac{3}{4} r^{-5 / 2} K_{1}\left(r, \lambda_{n}\right)\right) \\
& +\left(\frac{\partial}{\partial r}\right)\left(\frac{1}{\sqrt{r}} \frac{d^{2} K_{1}\left(r, \lambda_{n}\right)}{d r^{2}}\right. \\
& \left.\left.-r^{-3 / 2} \frac{d K_{1}\left(r, \lambda_{n}\right)}{d r}+\frac{3}{4} r^{-5 / 2} K_{1}\left(r, \lambda_{n}\right)\right)\right)
\end{aligned}
$$

Briefly, we consider the final form of Eq. (34a) to Eq. (34c) as:

$$
\begin{aligned}
&\left(\ddot{S}(t)+\left(\frac{\lambda_{n}}{\gamma}\right)^{2} S(t)\right)=-A A\left(b_{m} \bar{\Phi}_{1}+P(t)\right) \\
&-B B\left(E_{p} \bar{\Psi}_{1}+X(t)\right) \\
&\left(\frac{\Lambda}{\rho C_{V}} \zeta_{m}^{2} P(t)+\dot{P}(t)\right) N_{m}+C C P(t)^{2} N_{m} \\
&-D D P(t)^{2}-E E\left(E_{P} \bar{\Psi}_{1}\left(t, \gamma_{p}\right)+X(t)\right) P(t) \\
& \quad F F\left(a_{n} \bar{\omega}_{1}\left(t, \lambda_{n}\right)+S(t)\right) P(t) \\
&=H H\left(E_{p} \bar{\Psi}_{1}\left(t, \gamma_{p}\right)+X(t)\right)^{2} \\
&-K K P(t)\left(a_{n} \dot{\bar{\omega}}_{1}\left(t, \lambda_{n}\right)+\dot{S}(t)\right) \\
&- L L\left(a_{n} \bar{\omega}_{1}\left(t, \lambda_{n}\right)+S(t)\right)^{2} \\
&+ M M\left(a_{n} \bar{\omega}_{1}\left(t, \lambda_{n}\right)+S(t)\right)^{2} \\
&+ N N P(t)\left(E_{P} \bar{\Psi}_{1}\left(t, \gamma_{p}\right)+X(t)\right) \frac{1}{\rho C} N_{m} \\
& H P(t)\left(a_{n} \bar{\omega}_{1}\left(t, \lambda_{n}\right)+S(t)\right) \\
&\left(D \gamma_{p}^{2} F(t)+\dot{F}(t)\right) O_{p}=O O\left(b_{m} \bar{\Phi}_{1}\left(t, \zeta_{m}\right)+P(t)\right)
\end{aligned}
$$

$$
\begin{array}{cr}
+W\left(a_{n} \bar{\omega}_{1}\left(t, \lambda_{n}\right)+S(t)\right) & H P(t)\left(a_{n} \bar{\omega}_{1}\left(t, \lambda_{n}\right)+S(t)\right), \\
\cdot\left(\frac { 2 } { r } \left(\frac{d^{2} K_{1}\left(r, \lambda_{n}\right)}{d r^{2}}-r^{-1} \frac{d K_{1}\left(r, \lambda_{n}\right)}{d r}\right.\right. & \left(D \gamma_{p}^{2} F(t)+\dot{F}(t)\right) O_{p}=O O\left(b_{m} \bar{\Phi}_{1}(t, \zeta\right. \\
& +Q Q\left(a_{n} \bar{\omega}_{1}\left(t, \lambda_{n}\right)+S(t)\right) .
\end{array}
$$$$
\left.\left.+\frac{3}{4} r^{-2} K_{1}\left(r, \lambda_{n}\right)\right)\right)
$$$$
+\sqrt{r}\left(\frac{\partial}{\partial r}\right)\left(\frac{1}{\sqrt{r}} \frac{d^{2} K_{1}\left(r, \lambda_{n}\right)}{d r^{2}}\right.
$$$$
\left.-r^{-3 / 2} \frac{d K_{1}\left(r, \lambda_{n}\right)}{d r}+\frac{3}{4} r^{-5 / 2} K_{1}\left(r, \lambda_{n}\right)\right) .
$$

Also, the orthogonal property according to [48] is used:

$$
\begin{aligned}
& \int_{a}^{b} r K_{1}\left(r, \lambda_{n}\right) K_{1}\left(r, \lambda_{q}\right) d r=M_{n} \delta_{n q}, \\
& \int_{a}^{b} r K_{2}\left(r, \zeta_{m}\right) K_{1}\left(r, \zeta_{q}\right) d r=N_{m} \delta_{m q}, \\
& \int_{a}^{b} r K_{3}\left(r, \gamma_{p}\right) K_{3}\left(r, \gamma_{q}\right) d r=O_{p} \delta_{p q},
\end{aligned}
$$

where $\delta_{i j}$ is the Kronecher delta.

Constant coefficients are as follows:

$$
\begin{aligned}
& A A=\left(\frac{1}{M_{n} \gamma^{2}}\left(\frac{3 \lambda+2 \mu}{\lambda+2 \mu}\right) \alpha_{T} \int_{R_{i}}^{R_{o}} r K_{1}\left(t, \lambda_{n}\right)\right. \\
&\left.\left(\frac{d K_{2}\left(r, \zeta_{m}\right)}{d r}-\frac{K_{2}\left(r, \zeta_{m}\right)}{2 r}\right) d r\right), \\
& B B=\left(\frac{1}{M_{n} \gamma^{2}}\left(\frac{3 \lambda+2 \mu}{\lambda+2 \mu}\right) S_{g} \alpha_{C} \int_{R_{i}}^{R_{o}} r K_{1}\left(t, \lambda_{n}\right)\right. \\
&\left.\left(\frac{d K_{3}\left(r, \gamma_{P}\right)}{d r}-\frac{K_{3}\left(r, \gamma_{P}\right)}{2 r}\right) d r\right), \\
& C C= \frac{A}{\rho C}\left(\frac{1}{\sqrt{r}} \frac{d^{2} K_{2}\left(r, \zeta_{m}\right)}{d r^{2}}+r^{-3 / 2} \frac{d K_{2}\left(r, \zeta_{m}\right)}{d r}\right. \\
&\left.-\frac{1}{4} r^{-5 / 2} K_{2}\left(r, \zeta_{m}\right)\right),
\end{aligned}
$$




$$
\begin{aligned}
& D D=\int_{R_{i}}^{R_{o}} r K_{2}\left(r, \zeta_{m}\right) \frac{B}{\rho C}\left(\frac{d K_{2}\left(r, \zeta_{m}\right)}{d r}\right. \\
& \left.-r^{-3 / 2} \frac{d K_{1}\left(r, \lambda_{n}\right)}{d r}+\frac{3}{4} r^{-5 / 2} K_{1}\left(r, \lambda_{n}\right)\right)^{2} d r, \\
& \left.-\frac{1}{2} r^{-1} K_{2}\left(r, \zeta_{m}\right)\right)^{2} d r \\
& E E=\int_{R_{i}}^{R_{o}} r I K_{2}\left(r, \zeta_{m}\right) \frac{1}{\rho C}\left(\frac{d K_{2}\left(r, \zeta_{m}\right)}{d r}\right. \\
& \left.-\frac{1}{2} r^{-1} K_{2}\left(r, \zeta_{m}\right)\right)\left(\frac{1}{\sqrt{r}} \frac{d K_{3}\left(r, \gamma_{p}\right)}{d r}\right. \\
& \left.-\frac{1}{2} r^{-3 / 2} K_{3}\left(r, \gamma_{p}\right)\right) d r \\
& F F=\int_{R_{i}}^{R_{o}} r J K_{2}\left(r, \zeta_{m}\right) \frac{1}{\rho C}\left(\frac{d K_{2}\left(r, \zeta_{m}\right)}{d r}\right. \\
& \left.-\frac{1}{2} r^{-1} K_{2}\left(r, \zeta_{m}\right)\right)\left(\frac{1}{\sqrt{r}} \frac{d K_{3}\left(r, \gamma_{p}\right)}{d r}\right. \\
& \left.-\frac{1}{2} r^{-3 / 2} K_{3}\left(r, \gamma_{p}\right)\right)\left(\frac{1}{\sqrt{r}} \frac{d^{2} K_{1}\left(r, \lambda_{n}\right)}{d r^{2}}\right. \\
& N N=\int_{R_{i}}^{R_{o}} \frac{G}{\rho C} \frac{1}{\sqrt{r}} K_{2}\left(r, \zeta_{m}\right)\left(\frac{d^{2} K_{3}\left(r, \gamma_{p}\right)}{d r^{2}}\right. \\
& \left.+\frac{1}{r} \frac{d K_{3}\left(r, \gamma_{p}\right)}{d r}-\frac{1}{4 r^{2}} K_{3}\left(r, \gamma_{p}\right)\right) d r, \\
& O O=\int_{R_{i}}^{R_{o}} Z\left(-\zeta_{m}^{2} K_{2}\left(r, \zeta_{m}\right) K_{2}\left(r, \zeta_{m}\right) r K_{3}\left(r, \gamma_{p}\right)\right) d r, \\
& Q Q=\int_{R_{i}}^{R_{o}} W r K_{3}\left(r, \gamma_{p}\right)\left(\frac { 2 } { r } \left(\frac{d^{2} K_{1}\left(r, \lambda_{n}\right)}{d r^{2}}\right.\right. \\
& \left.-r^{-1} \frac{d K_{1}\left(r, \lambda_{n}\right)}{d r}+\frac{3}{4} r^{-2} K_{1}\left(r, \lambda_{n}\right)\right) \\
& +\sqrt{r}\left(\frac{\partial}{\partial r}\right)\left(\frac{1}{\sqrt{r}} \frac{d^{2} K_{1}\left(r, \lambda_{n}\right)}{d r^{2}}\right. \\
& \left.\left.-r^{-3 / 2} \frac{d K_{1}\left(r, \lambda_{n}\right)}{d r}+\frac{3}{4} r^{-5 / 2} K_{1}\left(r, \lambda_{n}\right)\right)\right) d r .
\end{aligned}
$$$$
\left.-r^{-3 / 2} \frac{d K_{1}\left(r, \lambda_{n}\right)}{d r}+\frac{3}{4} r^{-5 / 2} K_{1}\left(r, \lambda_{n}\right)\right) d r
$$$$
K K=\int_{R_{i}}^{R_{o}} \frac{D K_{2}\left(r, \zeta_{m}\right)}{\rho C}\left(\frac{1}{\sqrt{r}} \frac{d K_{1}\left(r, \lambda_{n}\right)}{d r}\right.
$$$$
\left.-\frac{1}{2} r^{-3 / 2} K_{1}\left(r, \lambda_{n}\right)\right) d r
$$$$
L L=\int_{R_{i}}^{R_{o}} \frac{E}{\rho C}\left(\frac{d^{2} K_{1}\left(r, \lambda_{n}\right)}{d r^{2}}-r^{-1} \frac{d K_{1}\left(r, \lambda_{n}\right)}{d r}\right.
$$$$
\left.+\frac{3}{4} r^{-2} K_{1}\left(r, \lambda_{n}\right)\right)\left(\frac{1}{\sqrt{r}} \frac{d K_{1}\left(r, \lambda_{n}\right)}{d r}\right.
$$$$
\left.-\frac{1}{2} r^{-3 / 2} K_{1}\left(r, \lambda_{n}\right)\right) d r
$$$$
M M=\int_{R_{i}}^{R_{o}} \frac{F}{\rho C} \sqrt{r}\left(\frac{1}{\sqrt{r}} \frac{d^{2} K_{1}\left(r, \lambda_{n}\right)}{d r^{2}}\right.
$$

Solving the equations above results in $S(t), P(t)$, and $X(t)$. Then, it is possible to obtain $\omega_{2}(r, t)$, $\Phi_{2}(r, t)$, and $\Psi_{2}=(r, t)$. Now, we have three ordinary equations, which should be solved simultaneously by some mathematical manipulations. Therefore, we have:

$$
\begin{aligned}
u(r, t)= & \frac{1}{\sqrt{r}} \sum_{n=1}^{\infty} a_{n} K_{1}\left(r, \lambda_{n}\right) \bar{\omega}_{1}\left(t, \lambda_{n}\right) \\
& +\sum_{n=1}^{\infty} S(t) K_{1}\left(r, \lambda_{n}\right), \\
T(r, t)= & \frac{1}{\sqrt{r}} \sum_{n=1}^{\infty} b_{m} K_{2}\left(r, \zeta_{m}\right) \bar{\Phi}_{1}\left(t, \zeta_{m}\right) \\
& +\sum_{n=1}^{\infty} P(t) K_{2}\left(r, \zeta_{m}\right), \\
C(r, t)= & \frac{1}{\sqrt{r}} \sum_{n=1}^{\infty} E_{p} K_{3}\left(r, \gamma_{p}\right) \bar{\Psi}_{1}\left(t, \gamma_{p}\right) \\
& +\sum_{n=1}^{\infty} X(t) K_{3}\left(r, \gamma_{p}\right) .
\end{aligned}
$$


Consequently, the triple coupled thermo-diffusemechanical equations are analytically solved using the discussed method. It is a general solution, which can be developed for any kind of boundary condition. It is only sufficient to have the kernel of transformation to obtain the response of triple coupled boundary value problems.

\section{Case study}

In this study, the quasi-static thermoelasticity problem of a pressurized sphere is analytically solved in which material properties vary with radial position. The axisymmetric equations of uncoupled thermoelasticity are:

$$
\begin{aligned}
& \frac{\partial^{2} T}{\partial r^{2}}+\frac{2}{r} \frac{\partial T}{\partial r}-\frac{\rho C_{V}}{\Lambda} \frac{d T}{d t}=0, \\
& \frac{\partial^{2} u}{\partial r^{2}}+\frac{2}{r} \frac{\partial u}{\partial r}-\frac{2 u}{r^{2}}=\frac{\alpha(1+v)}{(1-v)} \frac{\partial T}{\partial r} .
\end{aligned}
$$

Constitutive law states that the relation between stress components, radial displacement component, and temperature is as follows:

$$
\begin{aligned}
\sigma_{r r} & =\frac{E}{(1+v)(1-2 v)}\left((1-v) \frac{d u}{d r}+2 v \frac{u}{r}-(1+v) \alpha T\right), \\
\sigma_{\theta \theta} & =\frac{E}{(1+v)(1-2 v)}\left(v \frac{d u}{d r}+\frac{u}{r}-(1+v) \alpha T\right) .
\end{aligned}
$$

The analytical solution presented in the previous section was applied to a thick hollow sphere of the inner radius, $R_{i}=1 \mathrm{~m}$, and outer radius, $R_{O}=2 \mathrm{~m}$. Internal pressure exerted in inner surface of the hollow sphere was $\sigma_{i}=100 \mathrm{MPa}$, and outer surface was assumed to be traction-free. The thermal boundary conditions are as follows:

$$
\begin{aligned}
& \sigma\left(R_{i}, t\right)=100 \quad \mathrm{MPa}, \\
& \sigma\left(R_{O}, t\right)=0 .
\end{aligned}
$$

The material properties of sphere are shown in Table 1.

Solving the problem, the results are shown graphically in the following. Distribution of radial stress through the radial position is shown in Figure 2 for various instances of time. It can be seen that

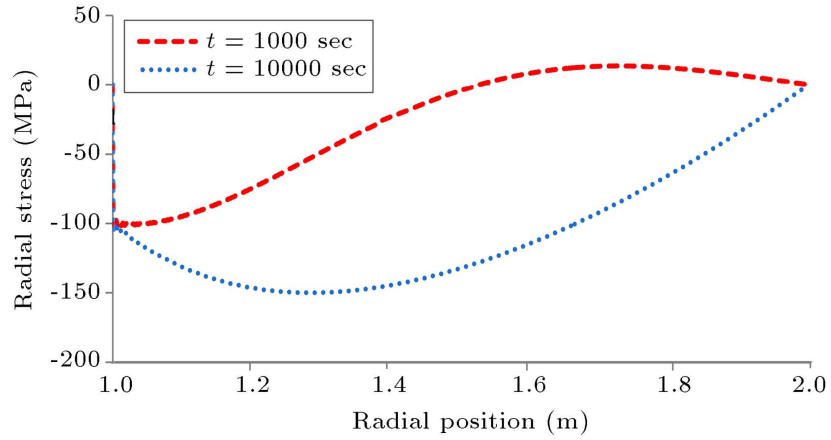

Figure 2. Distribution of radial stress through the radial position.

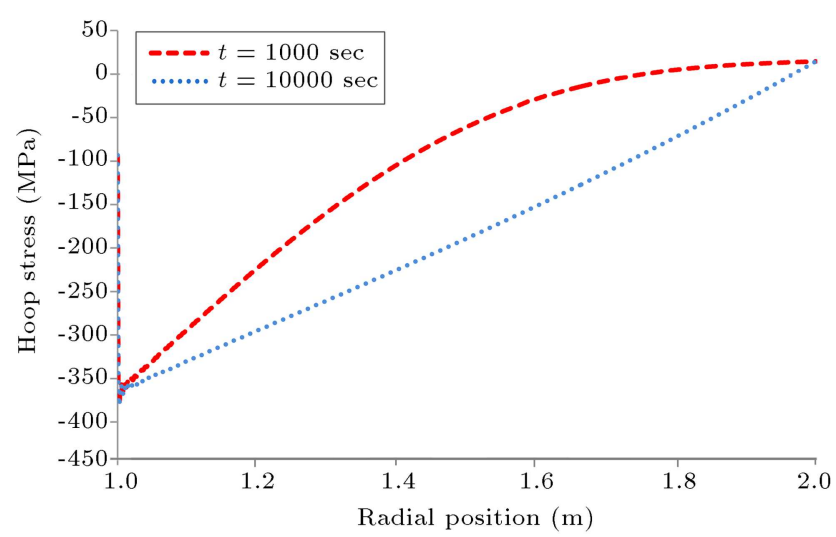

Figure 3. Distribution of hoop stress through the radial position.

radial stress decreases with time so that it becomes compressive as time grows up.

Figure 3 shows the distribution of hoop stress versus radial position for various instances of time. It is obvious that as time grows up, hoop stress becomes compressive in all positions.

Temperature distribution through the thickness of sphere is shown in Figure 4 for four time instances.

\section{Validation}

A spherically isotropic elastic hollow sphere problem is chosen as a case study. The problem solved is similar to the thermomechanical problem proposed by Wang et al. (2003). For symmetric problems, only the radial displacement is considered. Material properties are:

$$
E=200 \mathrm{GPa}, \quad v=0.3, \quad \alpha=10^{-6} \frac{1}{\mathrm{~K}} .
$$

Table 1. Material properties of sphere.

\begin{tabular}{cccccc}
\hline $\begin{array}{c}\text { Poisson's } \\
\text { ratio }\end{array}$ & $\begin{array}{c}\text { Elastic } \\
\text { modulus }\end{array}$ & Density & $\begin{array}{c}\text { Thermal } \\
\text { conductivity }\end{array}$ & $\begin{array}{c}\text { Specific } \\
\text { heat }\end{array}$ & $\begin{array}{c}\text { Coefficient of } \\
\text { thermal expansion }\end{array}$ \\
\hline$\nu$ & $E(\mathrm{GPa})$ & $\rho\left(\mathrm{kg} / \mathrm{m}^{3}\right)$ & $\Lambda(\mathrm{W} / \mathrm{mK})$ & $C_{V}(\mathrm{~J} / \mathrm{kg} \cdot \mathrm{K})$ & $\alpha_{T}\left(1 / \mathrm{K}^{\circ}\right)$ \\
0.3 & 70 & 2707 & 204 & 903 & $23 \times 10^{-6}$ \\
\hline
\end{tabular}




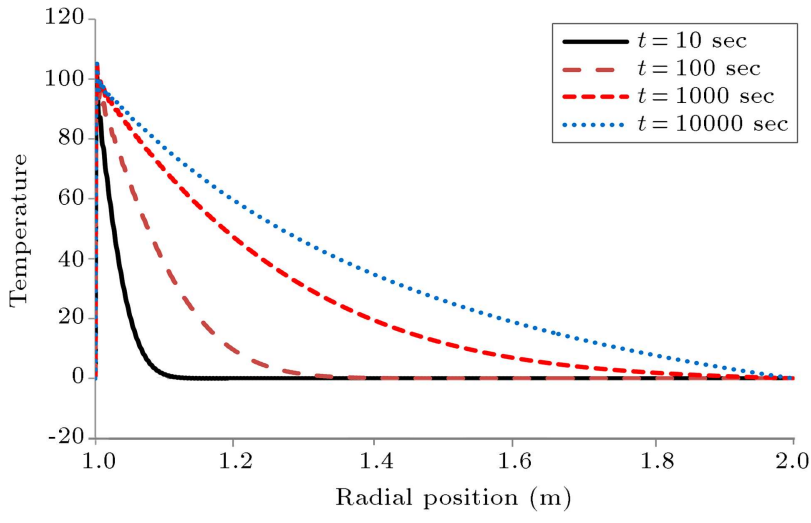

Figure 4. Distribution of temperature through thickness at different times.

Also, the boundary and initial conditions are as follows: Mechanical boundary and initial conditions are:

$$
\begin{aligned}
& \sigma_{r r}\left(R_{O}, t\right)=\sigma_{r r}\left(R_{i}, t\right)=0, \\
& u(r, t)=u(r, 0)=0 .
\end{aligned}
$$

Thermal boundary and initial conditions are of the following form:

$$
\begin{aligned}
& T\left(R_{O}, t\right)=T\left(R_{i}, t\right)=100, \quad K=T_{0}, \\
& T(r, 0)=0 .
\end{aligned}
$$

Mechanical stress for isotropic material is obtained by:

$$
\begin{aligned}
\sigma_{r r}(r, t)= & \frac{E}{(1+v)(1-2 v)}\left((1-v) \frac{\partial u(r, t)}{\partial r}\right. \\
& \left.+2 \frac{u(r, t)}{r}-\alpha(1+v) T(r, t)\right) \\
\sigma_{\theta \theta}(r, t)= & \frac{E}{(1+v)(1-2 v)}\left(v \frac{\partial u(r, t)}{\partial r}+\frac{u(r, t)}{r}\right) \\
& -\frac{\alpha E}{(1-2 v)} T(r, t)
\end{aligned}
$$

Substituting Eqs. (51a) and (51b) into Eq. (53a) results in:

$$
\begin{aligned}
& \frac{\partial u\left(R_{i}, t\right)}{\partial r}+\frac{2 v u\left(R_{i}, t\right)}{R_{i}(1-v)}=\frac{\alpha E(1+v)}{(1-v)} T_{0}, \\
& \frac{\partial u\left(R_{o}, t\right)}{\partial r}+\frac{2 v u\left(R_{o}, t\right)}{R_{o}(1-v)}=0 .
\end{aligned}
$$

Obviously, the boundary conditions above are of Cauchy type.
On the other hand, using the change of variables according to Eqs. (20a), (20b), and (20c), we have:

$$
\begin{aligned}
& \frac{\partial \omega\left(R_{i}, t\right)}{\partial r}+\frac{5 v-1}{2 R_{i}(1-v)} \omega\left(R_{i}, t\right)=\frac{\alpha \sqrt{R_{i}}(1+v)}{(1-v)} T_{0} \\
& \frac{\partial \omega\left(R_{o}, t\right)}{\partial r}+\frac{5 v-1}{2 R_{o}(1-v)} \omega\left(R_{o}, t\right)=0
\end{aligned}
$$

where initial conditions are:

$$
\begin{aligned}
& \omega(r, 0)=0, \\
& \dot{\omega}(r, 0)=0 .
\end{aligned}
$$

Also, a similar procedure is performed for Dirichlet boundary and initial conditions:

$$
\begin{aligned}
& \dot{\omega}(r, 0)=0, \\
& \Phi\left(R_{i}, t\right)=T_{0} \sqrt{R_{i}}, \\
& \Phi\left(R_{o}, t\right)=T_{0} \sqrt{R_{o}}, \\
& \Phi(r, 0)=0 .
\end{aligned}
$$

Eqs. (19) and (20) are rewritten for two coupled mechanical and thermal problems; then, the following relations are obtained:

$$
\begin{gathered}
\frac{\partial^{2} \omega}{\partial r^{2}}+\frac{1}{r} \frac{\partial \omega}{\partial r}-\frac{9}{4 r^{2}} \omega-\rho \frac{(1+v)(1-2 v)}{E(1-v)} \omega \\
-\left(\frac{1+v}{1-v}\right) \alpha_{T}\left(\frac{\partial \Phi}{\partial r}-\frac{\Phi}{2 r}\right)=0, \\
\frac{\partial^{2} \Phi}{\partial r^{2}}+\frac{1}{r} \frac{\partial \Phi}{\partial r}-\frac{1}{4 r^{2}} \Phi-\frac{\rho C}{K} \frac{\partial \Phi}{\partial t}=0 .
\end{gathered}
$$

To solve the mechanical and thermal equations, i.e., Eqs. (57) and (58), Eqs. (34a) and (34b), are respectively, employed and the following relations are thus obtained:

$$
\begin{aligned}
\bar{\omega}_{1}\left(t, \lambda_{n}\right) & =\ell^{-1}\left\{\frac{-\frac{2}{\gamma^{2} \pi} \frac{\alpha \sqrt{R_{i}}(1+v)}{(1-v)} T_{0}}{S\left(S^{2}+\frac{\lambda_{n}^{2}}{\gamma^{2}}\right)}\right\} \\
& =-\frac{2 \alpha}{\pi} \frac{\sqrt{R_{i}}(1+v)}{\lambda_{n}^{2}(1-v)} T_{0}\left[1-\cos \left(\frac{\lambda_{n} t}{\gamma}\right)\right] \\
\bar{\Phi}_{1}\left(t, \xi_{m}\right) & =\ell^{-1}\left\{\frac{-\frac{2}{\pi} \sqrt{R_{i}} T_{0}}{\left(S+\frac{K}{\rho C_{V}} \xi_{m}^{2}\right)}\right\} \\
& =-\frac{2}{\pi \xi_{m}^{2}} \sqrt{R_{i}} T_{0}\left[1-e^{-\frac{K}{\rho C_{V}} \xi_{m}^{2} t}\right]
\end{aligned}
$$


Moreover, Eq. (40a) is rewritten for the two coupled physics:

$$
\left(\ddot{S}(t)+\left(\frac{\lambda_{n}}{\gamma}\right)^{2} S(t)\right)=A A\left(b_{m} \bar{\Phi}\right) .
$$

Obtaining $S(t)$ from Eq. (46a) leads to:

$$
\begin{aligned}
& S(t)=\ell^{-1}\left\{\frac{(s S(0)-\dot{S}(0))+b_{m} A A\left(\frac{K}{\rho C}\right) \prod(S)}{S^{2}+\left(\frac{\lambda_{n}}{\gamma}\right)^{2}}\right\} \\
& =-\frac{2}{\pi \xi_{m}^{2}} \sqrt{R_{i}} T_{0} A A\left\{\left(\frac{\gamma}{\lambda_{n}}\right)^{2}\right. \\
& \left.-\frac{e^{-\frac{K}{\rho C} \xi_{m}^{2} t}}{\left(\frac{K}{\rho C} \xi_{m}^{2}\right)^{2}+\left(\frac{\lambda_{n}}{\gamma}\right)^{2}}\right\} \\
& +\frac{2 \sqrt{R_{i}} T_{0} A A K \gamma}{\pi \rho C_{V} \lambda_{n}\left(\left(\frac{K}{\rho C} \xi_{m}^{2}\right)^{2}+\left(\frac{\lambda_{n}}{\gamma}\right)^{2}\right)} \sin \left(\frac{\lambda_{n}}{\gamma} t\right) \\
& +\frac{2}{\pi \xi_{m}^{2}} \sqrt{R_{i}} T_{0} A A\left\{\left(\frac{\gamma}{\lambda_{n}}\right)^{2}\right. \\
& \left.-\frac{e^{-\frac{K}{\rho C} \xi_{m}^{2} t}}{\left(\frac{K}{\rho C} \xi_{m}^{2}\right)^{2}+\left(\frac{\lambda_{n}}{\gamma}\right)^{2}}\right\} \cos \left(\frac{\lambda_{n}}{\gamma} t\right) .
\end{aligned}
$$

Using Eqs. (47a) and (47b), it is possible to obtain $\omega(r, t)$ and $\Phi(r, t)$ so that we can obtain $u(r, t)$ and $T(r, t)$, as follows:

$$
\begin{aligned}
T(r, t)= & \sum_{\xi_{m}}\left\{\frac{b_{m} \bar{\Phi} K_{2}\left(r, \xi_{m}\right)}{\sqrt{r}}\right\}, \\
u(r, t)= & \sum_{\xi_{m}}\left\{\frac{a_{n} \bar{\omega}_{1} K_{1}\left(r, \lambda_{n}\right)}{\sqrt{r}}\right\} \\
& +\sum_{\lambda_{n}} \sum_{\xi_{m}}\left\{\frac{S(t) K_{1}\left(r, \lambda_{n}\right)}{\sqrt{r}}\right\} .
\end{aligned}
$$

To obtain $u(r, t)$, it is required to solve Eq. (63) for $S(t)$ by applying the boundary conditions:

$$
\begin{aligned}
S(t)= & \sum_{\lambda_{n}} \sum_{\xi_{m}}\left(-{\frac{2}{\pi \xi_{m}}}^{2} \sqrt{R_{i}} T_{0} A A\left\{\left(\frac{\gamma}{\lambda_{n}}\right)^{2}\right.\right. \\
& \left.-\frac{e^{-\frac{K}{\rho C} \xi_{m}^{2} t}}{\left(\frac{K}{\rho C} \xi_{m}^{2}\right)^{2}+\left(\frac{\lambda_{n}}{\gamma}\right)^{2}}\right\}
\end{aligned}
$$

$$
\begin{aligned}
& +\frac{2 \sqrt{R_{i}} T_{0} A A K \gamma}{\pi \rho C_{V} \lambda_{n}\left(\left(\frac{K}{\rho C} \xi_{m}^{2}\right)^{2}+\left(\frac{\lambda_{n}}{\gamma}\right)^{2}\right)} \sin \left(\frac{\lambda_{n}}{\gamma} t\right) \\
& +\frac{2}{\pi \xi_{m}^{2}} \sqrt{R_{i}} T_{0} A A\left\{\left(\frac{\gamma}{\lambda_{n}}\right)^{2}\right. \\
& \left.-\frac{e^{-\frac{K}{\rho C} \xi_{m}^{2} t}}{\left(\frac{K}{\rho C} \xi_{m}^{2}\right)^{2}+\left(\frac{\lambda_{n}}{\gamma}\right)^{2}}\right\} \cos \left(\frac{\lambda_{n}}{\gamma} t\right) .
\end{aligned}
$$

Now, the radial stress can be determined using the obtained results for $u(r, t)$ and $T(r, t)$. The radial stress can then be normalized in order to compare it with the results presented by Wang et al. [49]. Normalized radial stress is presented as a function of normalized factors, which are defined by Wang et al. [49] as:

Non-dimensional time:

$$
t^{*}=\frac{t}{b-a} \sqrt{\frac{E(1-v)}{\rho}} .
$$

Non-dimensional radius:

$$
\xi=\frac{r-a}{b-a}
$$

Non-dimensional radial stress:

$$
\sigma_{r r}^{*}=\frac{(1-2 v) \sigma_{r r}}{E \alpha T_{0}},
$$

where inner and outer radii are represented, respectively, by $a$ and $b$.

Figure 5 demonstrates a comparison of nondimensional radial stress $\left(\sigma_{r r}^{*}\right)$ versus non-dimensional time $\left(t^{*}\right)$ obtained in this study with those presented by Wang et al. [49]. To better illustrate the comparison of the results, the values of nondimensional radial stress obtained in this study for two values of $\xi=0.75$ and

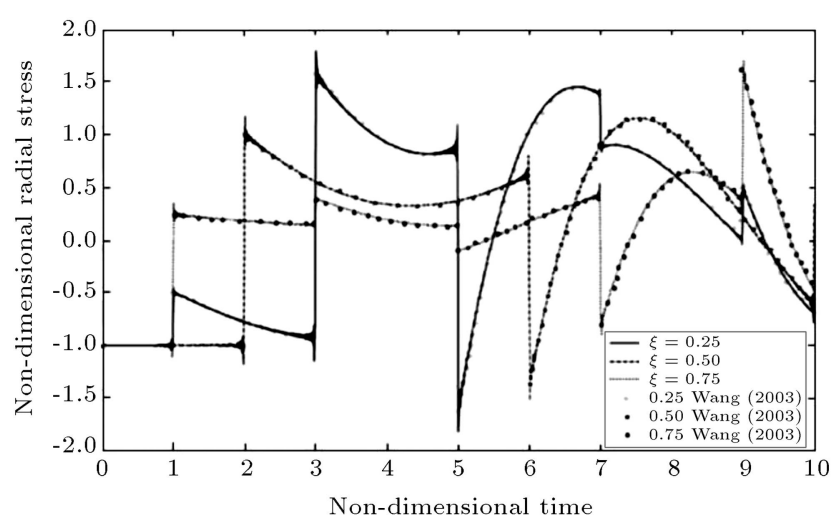

Figure 5. Variation of non-dimensional radial stress with time in different radial values for the isotropic hollow sphere. 
Table 2. Comparison of nondimensional radial stress for $\xi=0.75$ and $\xi=0.5$.

\begin{tabular}{ccc}
\hline Current study & Wang et al. [49] & Error (\%) \\
\hline \multicolumn{3}{c}{$\boldsymbol{\xi}=\mathbf{0 . 7 5}$} \\
0.25 & 0.26 & 0.04 \\
0.2 & 0.21 & 0.05 \\
0.18 & 0.17 & 0.06 \\
\hline 0.5 & $\boldsymbol{\xi}=\mathbf{0 . 5}$ \\
0.4 & 0.45 & 0.10 \\
0.3 & 0.4 & 0.00 \\
\hline
\end{tabular}

$\xi=0.5$ as well as those reported by Wang et al. [49] are tabulated in Table 1.

The results in Figure 5 and Table 2 show a good agreement between the solution developed using the method presented in the current study and the one proposed by Wang et al. [49].

\section{Conclusion}

The three coupled equations of mechanical stress, heat transfer, and diffusion were analytically solved using Hankel transform. The equations were derived based on the work of Rambert et al. [1] and the details of the derivation were given. The closed-form solution could provide a tool for an accurate calculation of the fields of mechanical stress-strain, temperature, and concentration. To validate this solution, the results of this study were compared with the results of dual coupled thermomechanical physics available in open literature and a good agreement was observed. For validating the performed solution to the dynamical problem of thermoelasticity, the problem of an isotropic sphere subjected to a sudden uniform temperature rise of $T_{0}$ throughout the sphere was considered. In other words, the entire sphere experienced a temperature rise of $T_{0}$. The presented solution approach to the boundary value problem provided a closed-form solution, which obviated the need for time-taking numerical methods or extensive experimental work to analyze the triple coupled mechanisms available in numerous industrial structures, such as gas and oil pipeline systems.

\section{Nomenclature}

$\begin{array}{ll}C & \text { Concentration } \\ C_{V} & \begin{array}{l}\text { Specific heat at constant strain and } \\ \text { normalized concentration }(\mathrm{J} / \mathrm{kg} . \mathrm{K})\end{array} \\ c_{g} & \text { Gas normalized concentration } \\ c_{p} & \text { Polymer normalized concentration }\end{array}$

D

D

d

E

f

J

$\bar{J}_{m g}$

$\bar{J}_{q}$

K

$k_{\mu}$

$k_{T}$

$P_{\text {in }}$

$q$

$r$

$s$

$S_{g}$

$T(X, t)$

$u$

$U$

$\dot{v}$

$W$

$C_{T \mu}$

$\boldsymbol{\alpha}(X, t)$

$\sigma$

$\rho$

$\Omega$

$\Omega_{0}$

$\lambda, \mu$

$\alpha_{C}$

$\alpha_{T}$

$\nu$

$\alpha_{i}(X, t)$

$\mu_{i}$

$t_{i}$

$\sigma_{i j} \quad$ Stress tensor $(\mathrm{Pa})$

$\varepsilon_{i j} \quad$ Strain tensor $\left(\mathrm{kg} / \mathrm{m}^{2} \mathrm{~s}\right)$

Kinematic energy (kg.s $/ \mathrm{m}^{3}$ )

Heat flux $(\mathrm{J} / \mathrm{s})$

Solubility $(1 / \mathrm{Pa})$

Displacement $(\mathrm{m})$

Work $\left(\mathrm{kg} / \mathrm{m}^{3}\right)$

Free energy

Initial energy $i$ th species

Traction
Defined as a function of coefficients $\rho$, $D$, and $k_{\mu}\left(\mathrm{m}^{2} / \mathrm{s}^{2}\right)$

Diffusivity $\left(\mathrm{m}^{2} / \mathrm{s}^{2}\right)$

Linking coefficient of effect of temperature variation (concentration variation) on the chemical potential

Young coefficient $(\mathrm{Pa})$

Body force per unit of mass $(\mathrm{N} / \mathrm{kg})$

Heat flux per unite area $\left(\mathrm{J} / \mathrm{m}^{2} . \mathrm{s}\right)$

Relative mass flux of the gas phase

Heat flux per unit area $\left(\mathrm{J} / \mathrm{m}^{2} \mathrm{~s}\right)$

Linking of the chemical potential gradient effect on the gas mass flux

Represents the thermal gradient effect on the entropy flux $\left(\mathrm{W} / \mathrm{m} . \mathrm{K}^{2}\right)$

Input power from the environment $(\mathrm{J})$

Volume density of heat generated by an outer source $\left(\mathrm{J} / \mathrm{m}^{3} . \mathrm{s}\right)$

Specific entropy $(\mathrm{J} / \mathrm{kgK})$

Absolute temperature (K)

Potential energy $(\mathrm{J})$

Acceleration $\left(\mathrm{m} / \mathrm{s}^{2}\right)$

Coupling coefficient corresponding to the temperature (kg.s $/ \mathrm{m}^{3}$ )

Internal state vector

Cauchy stress tensor $(\mathrm{Pa})$

Average density of the mixture

Lame elastic coefficients

Coefficient of mass transfer

Coefficient of thermal expansion $(1 / K)$

Poisson coefficient

Internal state variable

Mass chemical potential $(\mathrm{J} / \mathrm{kg})$ or the 


$\begin{array}{ll}\beta_{i j} & \text { Thermoelastic tensor } \\ \beta_{i j}^{\prime} & \text { Diffusion tensor } \\ T_{i j} & \text { Stress tensor }(\mathrm{Pa}) \\ C_{i j k l} & \text { Elastic tensor } \\ T r & \text { Trace operator } \\ \Gamma & \text { Area boundary } \\ \Omega & \text { Volume boundary } \\ \Lambda & \text { Thermal conductivity }(\mathrm{J} / \mathrm{m} . \mathrm{s} . \mathrm{K}) \\ \operatorname{div}[\boldsymbol{\square}] & \text { Divergence operator } \\ \text { Grad }\{\mathbf{\square}\} & \text { Gradient operator with respect to the } \\ & \text { reference }\end{array}$

$\operatorname{grad}\{\boldsymbol{\square}$ Gradient operator with refers to the current displacement

\section{References}

1. Rambert, G., Grandidier, J., Cangemi, L., and Meimon, Y. "A modelling of the coupled thermodiffusoelastic linear behaviour. Application to explosive decompression of polymers", Oil \& Gas Science and Technology, 58, pp. 571-591 (2003).

2. Vijalapura, P.K. and Govindjee, S. "Numerical simulation of coupled-stress case II diffusion in one dimension", Journal of Polymer Science Part B: Polymer Physics, 41, pp. 2091-2108 (2003).

3. Holalkere, V., Mirano, S., Kuo, A.-Y., Chen, W.T., Sumithpibul, C., and Sirinorakul, S. "Evaluation of plastic package delamination via reliability testing and fracture mechanics approach", 4 7th Conf. on Electronic Components and Technology, pp. 430-435 (1997).

4. Govindjee, S. and Simo, J.C. "Coupled stress-diffusion: Case II", Journal of the Mechanics and Physics of Solids, 41, pp. 863-887 (1993).

5. Busso, E. "Oxidation-induced stresses in ceramicmetal interfaces", Le Journal de Physique IV, 9, pp. Pr9-287-Pr9-296 (1999).

6. Klopffer, M. and Flaconneche, B. "Transport properdines of gases in polymers: bibliographic review", Oil \& Gas Science and Technology, 56, pp. 223-244 (2001).

7. Teh, L., Teo, M., Anto, E., Wong, C., Mhaisalkar, S., and Teo, P. "Moisture-induced failures of adhesive flip chip interconnects", Components and Packaging Technologies, IEEE Transactions on, 28, pp. 506-516 (2005).

8. Wahab, M.A., Ashcroft, I., Crocombe, A., and Shaw, S. "Diffusion of moisture in adhesively bonded joints", The Journal of Adhesion, 77, pp. 43-80 (2001).

9. Wong, E., Koh, S., Lee, K., and Rajoo, R. "Advanced moisture diffusion modeling and characterisation for electronic packaging", 52nd Conf. on Electronic Components and Technology, pp. 1297-1303 (2002).
10. Yu, Y.T. and Pochiraju, K. "Three-dimensional simulation of moisture diffusion in polymer composite materials", Polymer-Plastics Technology and Engineering, 42, pp. 737-756 (2003).

11. Shirangi, H., Auersperg, J., Koyuncu, M., Walter, H., Müller, W., and Michel, B. "Characterization of dualstage moisture diffusion, residual moisture content and hygroscopic swelling of epoxy molding compounds", Int. Conf. on Thermal, Mechanical and Multi-Physics Simulation and Experiments in Microelectronics and Micro-Systems, EuroSimE 2008, pp. 1-8 (2008).

12. Lin, T. and Tay, A. "Mechanics of interfacial delamination under hygrothermal stresses during reflow soldering", 1st Conf. on Electronic Packaging Technology, pp. 163-169 (1997).

13. Yi, S. and Sze, K.Y. "Finite element analysis of moisture distribution and hygrothermal stresses in TSOP IC packages", Finite Elements in Analysis and Design, 30, pp. 65-79 (1998).

14. Chang, K.-C., Yeh, M.-K., and Chiang, K.-N. "Hygrothermal stress analysis of a plastic ball grid array package during solder reflow", Proceedings of the Institution of Mechanical Engineers, Part C: Journal of Mechanical Engineering Science, 218, pp. 957-970 (2004).

15. Lahoti, S.P., Kallolimath, S.C., and Zhou, J. "Finite element analysis of thermo-hygro-mechanical failure of a flip chip package", 6th Int. Conf. on Electronic Packaging Technology, pp. 330-335 (2005).

16. Dudek, R., Walter, H., Auersperg, J., and Michel, B. "Numerical analysis for thermo-mechanical reliability of polymers in electronic packaging", 6th Int. Conf. on in Polymers and Adhesives in Microelectronics and Photonics, pp. 220-227 (2007).

17. Fan, X. and Zhao, J.-H. "Moisture diffusion and integrated stress analysis in encapsulated microelectronics devices", 12th Int. Conf. on in Thermal, Mechanical and Multi-Physics Simulation and Experiments in Microelectronics and Microsystems (EuroSimE), pp. 1-8 (2011).

18. Briscoe, B. and Liatsis, D. "Internal crack symmetry phenomena during gas-induced rupture of elastomers", Rubber Chemistry and Technology, 65, pp. 350-373 (1992).

19. Briscoe, B., Savvas, T., and Kelly, C. "Explosive decompression failure of rubbers: A review of the origins of pneumatic stress induced rupture in elastomers", Rubber Chemistry and Technology, 67, pp. 384-416 (1994).

20. Liatsis, D., Gas Induced Rupture of Elastomers, Imperial College London, London (1989).

21. Haghighi-Yazdi, M. and Lee-Sullivan, P. "Modeling of structural mechanics, moisture diffusion, and heat conduction coupled with physical aging in thin plastic plates", Acta Mechanica, 225, pp. 929-950 (2014).

22. McBride, A., Javili, A., Steinmann, P., and Bargmann, S. "Geometrically nonlinear continuum thermomechanics with surface energies coupled to diffusion", 
Journal of the Mechanics and Physics of Solids, 59, pp. 2116-2133 (2011).

23. Rambert, G. and Grandidier, J.-C. "An approach to the coupled behaviour of polymers subjected to a thermo-mechanical loading in a gaseous environment", European Journal of Mechanics-A/Solids, 24, pp. 151168 (2005).

24. Gibbs, J.W. "On the equilibrium of heterogeneous substances", American Journal of Science, pp. 441-458 (1878).

25. Gibbs, J.W. and Bumstead, H.A., Thermodynamics, 1, Longmans, Green and Company, pp. 1877-1961 (1906).

26. Duan, H., Wang, J., and Karihaloo, B.L. "Theory of elasticity at the nanoscale", Advances in Applied Mechanics, 42, pp. 1-68 (2009).

27. Miller, R.E. and Shenoy, V.B. "Size-dependent elastic properties of nanosized structural elements", Nanotechnology, 11, p. 139 (2000).

28. Mitrushchenkov, A., Chambaud, G., Yvonnet, J., and $\mathrm{He}, \mathrm{Q}$. "Towards an elastic model of wurtzite AlN nanowires", Nanotechnology, 21, pp. 255-702 (2010).

29. Yang, K., She, G.-W., Wang, H., Ou, X.-M., Zhang, X.-H., and Lee, C.-S. "ZnO nanotube arrays as biosensors for glucose", The Journal of Physical Chemistry C, 113, pp. 20169-20172 (2009).

30. Wei, G., Shouwen, Y., and Ganyun, H. "Finite element characterization of the size-dependent mechanical behaviour in nanosystems", Nanotechnology, 17, pp. 1118 (2006).

31. Yvonnet, J., Quang, H.L., and He, Q.-C. "An XFEM/level set approach to modelling surface/ interface effects and to computing the size-dependent effective properties of nanocomposites", Computational Mechanics, 42, pp. 119-131 (2008).

32. Gurtin, M.E. and Murdoch, A.I. "A continuum theory of elastic material surfaces", Archive for Rational Mechanics and Analysis, 57, pp. 291-323 (1975).

33. Angenent, S. and Gurtin, M.E. "Multiphase thermomechanics with interfacial structure 2. Evolution of an isothermal interface", Archive for Rational Mechanics and Analysis, 108, pp. 323-391 (1989).

34. Gurtin, M.E. and Matano, H. "On the structure of equilibrium phase transitions within the gradient theory of fluids", Quart. Appl. Math., 46, pp. 301-317 (1988).

35. Gurtin, M.E. and Struthers, A. "Multiphase thermomechanics with interfacial structure", Archive for Rational Mechanics and Analysis, 112, pp. 97-160 (1990).

36. Daher, N. and Maugin, G. "The method of virtual power in continuum mechanics application to media presenting singular surfaces and interfaces", Acta $\mathrm{Me}$ chanica, 60, pp. 217-240 (1986).

37. Dolbow, J., Fried, E., and Ji, H. "Chemically induced swelling of hydrogels", Journal of the Mechanics and Physics of Solids, 52, pp. 51-84 (2004).
38. Ji, B. and Gao, H. "Mechanical properties of nanostructure of biological materials", Journal of the Mechanics and Physics of Solids, 52, pp. 1963-1990 (2004).

39. Liu, C.-L., Ho, M.-L., Chen, Y.-C., Hsieh, C.-C., Lin, Y.-C., and Wang, Y.-H. "Thiol-functionalized gold nanodots: two-photon absorption property and imaging in vitro", The Journal of Physical Chemistry $C$, 113, pp. 21082-21089 (2009).

40. Steinmann, P. "On boundary potential energies in deformational and configurational mechanics", Journal of the Mechanics and Physics of Solids, 56, pp. 772-800 (2008).

41. Rodier Renaud, L. "Etude transitiometrique de l'influence de la pression sur les proprietes thermomecaniques des polymeres. Expansibilite thermique jusqu'a $393 \mathrm{k}$ et $300 \mathrm{mpa}$ de polyethylenes seuls ou en interaction avec le methane", Clermont Ferrand 2, (1994).

42. Cunat, C. "A thermodynamic theory of relaxation based on a distribution of non-linear processes", Journal of Non-Crystalline Solids, 131, pp. 196-199 (1991).

43. Fosdick R. and Tang, H. "Surface transport in continuum mechanics", Mathematics and Mechanics of Solids, 14(6), pp. 587-598 (2008).

44. Coleman, B.D. and Gurtin, M.E. "Thermodynamics with internal state variables", The Journal of Chemical Physics, 47, pp. 597-613 (1967).

45. Coleman, B.D. and Gurtin, M.E. "On the growth and decay of discontinuities in fluids with internal state variables", Physics of Fluids, 10(7), pp. 1454-1458 (1967).

46. Michaels, A.S. and Bixler, H.J. "Flow of gases through polyethylene", Journal of Polymer Science, 50, pp. 413-439 (1961).

47. Michaels, A.S. and Bixler, H.J. "Solubility of gases in polyethylene," Journal of Polymer Science, 50, pp. 393-412 (1961).

48. Sneddon, I.N., The Use of Integral Transforms, McGraw-Hill Book Company, New York (1972).

49. Wang, H., Ding, H., and Chen, W. "Transient responses in a piezoelectric spherically isotropic hollow sphere for symmetric problems", Journal of Applied Mechanics, 70, pp. 436-445 (2003).

\section{Biographies}

Ehsan Mahdavi is a PhD student in the Department of Mechanical Engineering at University of Tehran, Iran. He received his MSc degree from the Department of Mechanical Engineering at Khaje Nasir University of Technology. His main areas of research include noncomposite and composite materials, and viscoelasticity.

Mojtaba Haghighi-Yazdi received his BSc and MSc degrees in the field of Mechanical Engineering from Iran 
University of Science and Technology. His MSc thesis focused on fatigue modeling of composite laminates with stress concentration. He earned his $\mathrm{PhD}$ in the field of Mechanical Engineering from University of Waterloo. His $\mathrm{PhD}$ thesis centered around modeling of the four-coupled physics available in hygrothermal aging of a polymer blend. He has been with University of Tehran as a faculty member since 2012 and his current research interests include composite materials, mechanical behavior of polymeric materials, and viscoelasticity.

Majid Baniassadi is an Assistant Professor in the School of Mechanical Engineering, University of Tehran, Iran. He holds a PhD degree in Mechanics of Materials from the University of Strasbourg, received in 2011. He received his MSc degree from the University of Tehran in 2007 and BSc degree from Isfahan University of Technology in 2004, both in Mechanical Engineering. His research interests include multiscale analysis and micromechanics of heterogeneous materials, numerical methods in engineering, and electron microscopy image processing for microstructure identification.

Mehran Tehrani is an Assistant Professor of Mechanical Engineering at the University of New Mexico (UNM). He received his $\mathrm{PhD}$ degree in Engineering Mechanics from Virginia Tech in 2012. He is currently the Director of the Advanced Structural and Energy Materials Laboratory (ASEMlab) at UNM.

Saïd Ahzi is currently a Research Director of the
Materials Science and Engineering Group at Qatar Environment and Energy Research Institute (QEERI) and Professor in the College of Science \& Engineering, Hamad Bin Khalifa University, Qatar Foundation, Qatar. He holds a position as a Distinguished Professor at the University of Strasbourg (Exceptional Class). He also holds an Adjunct Professor position with the School of Materials Science and Engineering at Georgia Institute of Technology, USA. In January 2000, he joined the Faculty of Physics and Engineering at the University of Strasbourg, France, as Full Professor. From 1995 to 2000, he held the position of Professor (assistant, then associate) in the Department of Mechanical Engineering at Clemson University, USA. Prior to this, he spent four years as a Research Scientist and Lecturer at the University of California in San Diego, USA, and four years as a Postdoctoral Research Associate at Massachusetts Institute of Technology, USA. From 2007 to 2011, he held an Adjunct Research Professor position with the University of Aveiro, Portugal. Dr. Saïd Ahzi advised about 25 PhDs and 24 Master's degrees, and was the scientific advisor for 6 habilitations. He published more than 250 scientific papers in the areas of materials science, applied mechanics, and processing.

Jalil Jamali is an Assistant Professor of Mechanical Engineering at Islamic Azad University of Shoushtar. $\mathrm{He}$ received his $\mathrm{PhD}$ degree in Engineering Mechanics from University of Tehran. His main areas of research include elastodynamics, acoustic, nanomechanics, fluid mechanics, dynamics, vibrations, and elasticity. 